Rev. Asoc. Esp. Neuropsiq. 2020; 40(I 37): I 5 5-202

DOI: I0.432 I/So2 I I-57352020000I00009

\title{
TAT: Normas aperceptivas en pacientes institucionalizados con trastorno mental grave y prolongado
}

TAT: Apperceptive norms in hospitalized patients with severe and prolonged mental disorders

\author{
Enrique Bermúdez Navas ${ }^{a}$, Emilio González-Pablos ${ }^{b}$, Rosa Sanguino \\ Andrésci, Daniel San Segundo Rivera ${ }^{d}$ \\ a) Médico psiquiatra en Centro Asistencial San Juan de Dios, Palencia. \\ b) Médico psiquiatra en Hermanas Hospitalarias Centro Sociosanitario, Palencia. \\ c) Psicóloga clínica en Centro Asistencial Universitario de Palencia. \\ d) Médico especialista en Medicina Interna en Centro Asistencial Universitario de Palencia.
}

Correspondencia: Enrique Bermúdez Navas (enrique.bn@hotmail.com)

Recibido: 15/10/2019; aceptado con modificaciones: 3/4/2020

Resumen: El Test de Apercepción Temática (TAT) es un test proyectivo inventado en 1935. Repasamos su origen, fundamento y utilidad a través del uso que del test han realizado diversos autores a lo largo de su historia. Asimismo, revisamos sus aplicaciones más recientes y reflexionamos sobre sus posibilidades. Por último, replicamos el estudio del que se sirvió Saul Rosenzweig para establecer normas de apercepción temática que se disponen en población general. En el presente estudio, con una metodología más rigurosa que la descrita por Rosenzweig, sugerimos un patrón de respuesta al test en sujetos con trastorno mental grave (fundamentalmente, esquizofrenia) que podría emplearse en análisis actuales de las respuestas al test.

Palabras clave: Test de Apercepción Temática, test proyectivos, personas con trastorno mental grave, esquizofrenia. 


\begin{abstract}
The Thematic Apperception Test (TAT) is a projective test created in 1935. In this article, its origin, bases and the usefulness that has shown throughout its history are reviewed. In addition, we examine its more recent applications and reflect on its possibilities. Finally, we replicate the study conducted by Saul Rosenzweig in order to establish adult apperceptive norms in the general population. In this paper, with a more rigorous methodology than that of Rosenzweig, we suggest a response pattern in individuals with severe mental disorders (mostly, schizophrenia).
\end{abstract}

Key words: Thematic Apperception Test, projective tests, people with severe mental disorders, schizophrenia.

\title{
INTRODUCCIÓN
}

Elas L Test de Apercepción Temática (TAT) es un test proyectivo que aparece en na Morgan, aunque la serie de láminas que se emplea actualmente data de 1943 (1). La prueba consiste en mostrar al examinado una colección de láminas que reproducen escenas dramáticas que, por sus contornos imprecisos, su impresión difusa o su tema inexplícito, son lo suficientemente ambiguas como para provocar la proyección de la realidad íntima del sujeto a través de sus interpretaciones preceptúales, mnésicas e imaginativas (2).

Algunos psiquiatras españoles, como el catedrático Carlos Castilla del Pino, defendían la aplicación de este tipo de test al considerar que nos hacen ostensibles los factores más decididores de la motivación de la conducta y tienen la función de ampliar el campo de referencias del paciente en niveles que la mera entrevista apenas hubiera sido suficiente para dilucidar (3). Castilla del Pino también se sirve de las respuestas de sujetos al test para ilustrar y dar soporte a su teoría de los sentimientos (4).

Durante la realización de la prueba, el examinado tiende a ajustarse a ciertas tendencias y patrones regulares de respuesta a modo de normas que permiten diferenciar entre la producción esencial del individuo y la producción típica popular de su mismo grupo.

Partimos de la base de que la comparación de la producción de un sujeto con el grupo será más rigurosa si se cuenta con normas, dado que los enfoques psicométricos y proyectivos no son antagónicos sino complementarios, y que es preciso saber qué se considerará como respuesta individual y qué se considerará común o cliché.

Desde el manual para la aplicación del TAT, se promueve una tarea de normalización de las normas temáticas en otros países para obtener la máxima eficacia del TAT en beneficio de la clínica y de la investigación. Así, por ejemplo, las normas para 
adultos brasileños fueron obtenidas en 1953-55 en el Instituto de Selección y Orientación Profesional de Río de Janeiro sobre 500 adultos normales y marginales (5).

Saul Rosenzweig estableció en 1944-45 las normas aperceptivas y los tiempos de reacción, el tiempo total y el número total de palabras del relato para diecisiete láminas. La muestra de su estudio estaba integrada por 100 sujetos adultos norteamericanos (50 hombres y 50 mujeres) "normales" (este juicio se extraía mediante una breve historia de cada examinado en relación con trastornos nerviosos, tratamiento psiquiátrico o institucionalización, excluyendo del estudio a los sujetos con datos positivos), cuyas edades oscilaban entre los 20 y los 40 años (6).

En nuestro medio, el psicólogo clínico y catedrático Alejandro Ávila Espada redactó su tesis doctoral en 1983 sobre el TAT en población española. Realizó un estudio normativo en el que se incluyeron 100 sujetos ( 49 hombres y 51 mujeres) de entre 14 y 53 años (media de 25,76 años), la mayoría solteros, de clase socioeconómica y nivel educacional medio, procedentes de las distintas zonas geográficas españolas. Se excluyeron todos aquellos sujetos que contaran con algún antecedente personal o familiar de trastorno mental o manifestasen síntomas psicopatológicos durante el proceso de selección (7). El documento ofrece un extenso material, rico y detallado.

Muchos otros autores han trabajado con el test desde que se dispone de la prueba, tanto para explicar la fenomenología de la esquizofrenia y sus síntomas como para interactuar con conceptos contemporáneos como puede ser la cognición social. Estas investigaciones abarcan los síntomas de la esquizofrenia pero también trastornos relacionados, factores pronósticos y pruebas complementarias. Por citar algunos ejemplos, Jones describió reglas para medir el riesgo de desarrollar psicopatología esquizofreniforme en adultos jóvenes a partir de las respuestas al TAT de sus padres (8). Manschreck se sirvió del TAT para evaluar a personas con familiares con esquizofrenia (9). Varias investigaciones han estudiado los patrones de comunicación observados en las respuestas en el TAT de los padres de pacientes esquizofrénicos (1012) o el concepto de la madre esquizofrenógena y el TAT $(13,14)$. Waszkiewicz y Lysaker han establecido correlaciones entre los trastornos del lenguaje y las funciones cognitivas en personas con trastornos esquizofrénicos y los resultados de diversos test entre los que figuraba el TAT $(15,16)$. Kenchadze ha empleado el TAT para conocer la personalidad o los cambios en la misma generados por la progresión de la esquizofrenia, empleándose asimismo el test para observar los cambios que se producen tras tratamiento psicofarmacológico y el consumo de drogas $(17,18)$ y Fowler lo utilizó para monitorizar los resultados en psicoterapia (19). Otros se han aproximado a evaluar el riesgo suicida a partir de las respuestas en el TAT (20). Bouvet y Stein han aprovechado el material que ofrece el test para aplicar escalas con objeto de explorar la validez de constructo de las mismas, establecer comparaciones entre grupos o 
correlaciones con otras escalas relacionadas con el lenguaje, la comunicación y la cognición social $(21,22)$, entre otros tipos de análisis propuestos $(23-25)$.

Asimismo, cabe subrayar la utilidad del TAT en la actualidad, tanto en la práctica clínica como en investigación, pues se publican artículos novedosos en pleno siglo XXI que integran el TAT en la evaluación de la persona con esquizofrenia o se nutren del test para presentar hipótesis de trabajo sobre la psicosis. Por ejemplo, Steffen Moritz utilizó el TAT para estudiar los procesos de pensamiento en una muestra de pacientes con esquizofrenia, con el propósito de proporcionar información adicional sobre los sesgos que llevan a estos pacientes a extraer conclusiones precipitadas en base a una escasa evidencia en comparación con pacientes control (26).

El presente estudio, mediante la descripción de un posible patrón típico de producción de pacientes institucionalizados con trastorno mental grave y prolongado (la mayoría esquizofrenia) cuando se enfrentan a varias láminas del TAT, pretende servir de aproximación para que los examinadores, a la hora de administrar sus test, puedan disponer de unas referencias que sugieran normas en nuestro medio actual con las que establecer el ajuste de la persona con el grupo de trastorno mental grave y prolongado, además de determinar así lo que es característica individual o historia cliché dentro de este tipo de población.

A diferencia de lo ocurrido con el test de Rorschach, en las últimas décadas no ha habido muchas investigaciones que contribuyan específicamente a la estandarización del TAT (con datos normativos, etc.) y el ánimo de este estudio contribuye a paliar esta carencia. Se trata, pues, de un intento más por reactivar la literatura científica precedente, refrescando un test con amplias posibilidades.

\section{Material y MÉTODO}

Según el manual del TAT (27) y al no disponer de normas o tablas de frecuencias en este tipo de población, a fin de discriminar cuándo un examinador se halla ante lo desacostumbrado, el examinador ha de reunir, cuando menos, cincuenta o más repertorios de historias.

Basándonos en esta cuestión, en nuestro estudio se seleccionaron aleatoriamente 50 sujetos de entre todos los pacientes ingresados en la Unidad de Admisión del Área de Psiquiatría de Hermanas Hospitalarias Centro Sociosanitario en Palencia ( $54 \%$ hombres y $46 \%$ mujeres), excluyendo aquellos que no comprendieran las instrucciones de la prueba.

La muestra consistía en 50 sujetos con una edad media de 57,02 años (DE \pm 8,65), mediana 58 años (rango intercuartílico 25-75\% = 53,62 años). El nivel académico se distribuyó del siguiente modo: estudios secundarios (40\%), universitarios (34\%) y primarios (24\%). Los diagnósticos fueron: esquizofrenia (84\%), trastorno 
esquizoafectivo (6\%), trastorno bipolar (4\%), trastorno esquizofreniforme (2\%), trastorno obsesivo-compulsivo (2\%) y trastorno de personalidad (2\%). La cantidad de años de ingreso en el momento del estudio fue de entre 11 y 20 ańos (46,93\%); $\leq 10$ años (34,69\%); $y \geq 21$ años: $9(18,36 \%)$.

Todas las pruebas fueron suministradas y explicadas por el mismo examinador médico, que además llevó a cabo la organización de las respuestas en forma de tablas inspiradas en las normas de apercepción temática de Rosenzweig (división de cada lámina según figuras: sexo, edad, identificación y otras caracterizaciones; objetos; y problemas y soluciones), siempre que superasen una frecuencia de aparición del $10 \%$ (en lugar del 20\% que establecía Rosenzweig). Al igual que Rosenzweig, el examinador podía recurrir a la agrupación de varias respuestas para la creación de tablas.

Como preparación, antes de la prueba el examinador mantuvo al menos una o varias entrevistas de familiarización con cada paciente seleccionado, con el propósito de generar un espacio de respeto, comprensión y apoyo que permitiese ajustar los comentarios al estado emocional de cada sujeto previo a la realización de la prueba, para confirmar que los sujetos no se encontrasen en estado de descompensación aguda. Así trató de fomentarse la espontaneidad y facilitarse la recogida de datos.

Como consigna, se pedía a los sujetos que proporcionaran una historia que comprendiese el pasado (sucesos determinantes en la escena), presente (las acciones, pensamientos y sentimientos de los personajes) y futuro (desenlace) para el contenido de cada lámina, no dando a entender que las respuestas fuesen correctas o incorrectas. Entonces, se mostraban las láminas siguiendo siempre el mismo orden y se preguntaba con cada lámina mostrada "¿Qué sucede aquí?" (siguiendo la fórmula examinadora de Castilla del Pino), adoptando un papel neutro durante el desarrollo de las historias y tratando de evitar la intervención. En ningún caso se facilitó una historia de ejemplo.

Se emplearon 16 láminas en total. Los pacientes narraron historias para las siguientes láminas en el siguiente orden: lámina 1: El chico y el violín; lámina 2: La estudiante en el campo; lámina 3VH: Reclinado/a en el diván (solo hombres); lámina $3 \mathrm{NM:} \mathrm{La} \mathrm{joven} \mathrm{en} \mathrm{la} \mathrm{puerta} \mathrm{(solo} \mathrm{mujeres);} \mathrm{lámina} \mathrm{4:} \mathrm{Mujer} \mathrm{que} \mathrm{retiene} \mathrm{al}$ hombre; lámina $6 \mathrm{VH}$ : El hijo que se va (solo hombres); lámina $7 \mathrm{VH}$ : Padre e hijo (solo hombres); lámina $7 \mathrm{NM}$ : Niña y muñeca (solo mujeres); lámina $8 \mathrm{VH}$ : La intervención quirúrgica (solo hombres); lámina $9 \mathrm{NM}$ : Dos mujeres en la playa (solo mujeres); lámina 10: El abrazo; lámina $12 \mathrm{M}$ : La celestina (solo mujeres); lámina 13 HM: Mujer en la cama; lámina 14: Hombre en la ventana; lámina $18 \mathrm{VH}$ : Atacado desde atrás (solo hombres); lámina $18 \mathrm{NM:} \mathrm{Mujer} \mathrm{que} \mathrm{estrangula} \mathrm{(solo} \mathrm{mujeres).}$

Se establecieron los casos en los que se podía reclamar la intervención del examinador y la forma de proceder en caso de inadecuación a la consigna. Como norma general, se acentuaba y se repetía la consigna con cada incumplimiento y se intentaba que la intervención del examinador fuera lo más breve posible. Si el su- 
jeto pedía explicación acerca de algún objeto de las láminas, se le decía que "puede ser cualquier cosa, lo que usted prefiera o lo primero que se le ocurra". Si el sujeto presentaba dificultades de ideación, se podía formular una o varias de las siguientes preguntas secuencialmente: “QQué sucede?, ¿qué figuras aparecen?, ¿qué relación hay entre ellas?, ¿qué piensan o cómo se sienten?, ¿por qué motivo? y ¿qué pasa al final?". En caso de conformidad superficial, estaba permitido formular preguntas detalladas exclusivamente basadas en las manifestaciones anteriores del sujeto durante la narración. Si el sujeto vacilaba, se le pedía que decidiera entre las distintas posibilidades que narraba y continuase el argumento de la historia. Si persistía en la vaguedad, se le instaba a ser más específico o se le permitía narrar la primera historia que se le ocurriese. Se podía repetir en voz alta la última oración del sujeto en caso de lentitud indebida. No se produjo interrogatorio entre una lámina y la siguiente, ni tras la última lámina. Se permitía indagar en ambigüedades (por ejemplo, para diferenciar entre un lapsus linguae y una distorsión en el sexo de las figuras). En caso de omisiones, al final de la narración podía preguntarse "¿Algún otro detalle?". No se suspendió ninguna prueba por incumplimiento.

Las historias se registraron mediante la grabación con micrófono no oculto en una sola sesión con cada paciente. Posteriormente, se llevó a cabo una transcripción literal de los relatos, las preguntas y el comportamiento de los sujetos durante la prueba, así como de los comentarios del propio examinador. En el caso de siete pacientes no se pudo transcribir literalmente todo el discurso por mala vocalización o tono de voz muy bajo. A tres de las pacientes no se les presentó la lámina $3 \mathrm{NM}$ "La joven en la puerta" por olvido.

Se cronometró el tiempo que el sujeto se demoraba en comenzar su narración después de la presentación de cada lámina y el tiempo total desde la presentación de cada lámina hasta que concluía la historia.

El estudio fue aprobado por el Comité de Investigación y Biblioteca del Complejo Asistencial Universitario de Palencia y se llevó a cabo durante agosto de 2015.

Los participantes (o sus tutores legales en caso de incapacidad civil) otorgaron su consentimiento informado.

No se compensó a ningún paciente por su participación.

\section{AnÁlisis ESTADÍSTICO}

Los datos fueron analizados con el programa SPSS versión 24. Todas aquellas variables que no alcanzaran una aparición mínima del $10 \%$ no se contabilizaron en las tablas (figuran como "sin resultados"). Se realizaron análisis descriptivos de las medias y las desviaciones estándar para todas las láminas. Además de las medidas de frecuencia, se realizaron comparaciones entre sexos para aquellas láminas comunes a hombres y 
mujeres, donde se estudiaron las variables sociodemográficas, los tiempos, el número de palabras y las respuestas al test. Para analizar las variables sociodemográficas y clínicas, atendiendo a la normalidad o no de la distribución, se empleó la prueba t de Student para los años de ingreso y el Mann-Whitney U test para comparar la edad, los años de ingreso y el nivel de estudios. En cuanto a los valores tiempo de reacción, tiempo total y número de palabras, se realizó el test de bondad de ajuste a la normalidad Shapiro-Wilk. Al no seguir la distribución normal, el valor que mejor definía la frecuencia para estos parámetros fue la mediana y la dispersión (percentiles 25 y 75) para los dos grupos. A continuación se determinó realizar pruebas no paramétricas para dos muestras independientes mediante el Mann-Whitney $\mathrm{U}$ test. Para comparar las respuestas al test (el contenido de las láminas), se realizó el test de Chi-cuadrado atendiendo a la razón de máxima verosimilitud y con prueba exacta de Fisher cuando lo necesitase.

\section{Resultados}

Como muestran las Tablas 1-3, no se encontraron diferencias significativas en cuanto a edad, nivel de estudios o diagnóstico entre hombres y mujeres. Sin embargo, como muestra la Tabla 4, sí se encontraron diferencias significativas en cuanto a los años de ingreso ( $\mathrm{p}=0,01)$ a favor de las mujeres (15,6 años) frente a los hombres (10,1 años).

TABla I

Edad

\begin{tabular}{|c|c|c|c|c|}
\hline \multicolumn{4}{|c|}{ Edad } & p \\
\cline { 1 - 3 } Hombres & \multicolumn{2}{c|}{ Mujeres } & \multirow{2}{*}{0,09} \\
\cline { 1 - 3 } Mediana & Pc 25 a 75 & Mediana & Pc 25 a 75 & \\
\cline { 1 - 3 } 56 & 52 a 56 & 60 & 55 a 64 & \\
\hline
\end{tabular}

TABLA 2

Nivel de estudios

\begin{tabular}{|c|c|c|c|c|}
\hline Nivel de estudios & Hombres & Mujeres & Total & $\mathrm{p}$ \\
\hline Primarios & $25,9 \%$ & $22,7 \%$ & $24,5 \%$ & 1 \\
\hline Secundarios & $40,7 \%$ & $40,9 \%$ & $40,8 \%$ & \\
\cline { 1 - 3 } Universitarios & $33,3 \%$ & $36,4 \%$ & $34,7 \%$ & \\
\hline
\end{tabular}


TABLA 3

Diagnóstico

\begin{tabular}{|l|c|c|c|c|}
\hline Diagnóstico & Hombres & Mujeres & Total & $\mathrm{p}$ \\
\hline Esquizofrenia & $88,9 \%$ & $78,3 \%$ & $84,0 \%$ & 0,2 \\
\hline Trastorno esquizofreniforme & $3,7 \%$ & $0,0 \%$ & $2 \%$ & \\
\hline Trastorno obsesivo-compulsivo & $3,7 \%$ & $0,0 \%$ & $2 \%$ & \\
\hline Trastorno esquizoafectivo & $0,0 \%$ & $13,0 \%$ & $6 \%$ & \\
\hline Trastorno bipolar & $3,7 \%$ & $4,3 \%$ & $4 \%$ & \\
\hline Trastorno de personalidad & $0,0 \%$ & $4,3 \%$ & $2 \%$ & \\
\hline
\end{tabular}

TABla 4

Años de ingreso

\begin{tabular}{|l|c|c|c|}
\hline Ańos de ingreso & Media & DE & $\mathrm{p}$ \\
\hline Hombres & 10,1 & 5,6 & $\mathbf{0 , 0 1}$ \\
\hline Mujeres & 15,6 & 8,3 & \\
\hline
\end{tabular}

A continuación, resumiremos las historias más frecuentes pertenecientes a los sujetos con trastorno mental grave y anotaremos algunas consideraciones que forzosamente deben concernir también a aquellos estudios que le han servido de referencia en población general.

La lámina con el mayor tiempo de reacción para los hombres es la lámina 13 -"Mujer en la cama"- (media de 11 segundos) y para las mujeres es la lámina 9 NM -"Dos mujeres en la playa"- (media de 11,52 segundos). Para ambos sexos, la lámina con el menor tiempo de reacción es la lámina 1 -"El niño y el violín"- (media de 3,56 segundos). En el caso de los hombres, coincide con los hallazgos de Rosenzweig. 
TABla 5

Tiempo de reacción

\begin{tabular}{|l|c|c|c|c|c|}
\hline \multirow{2}{*}{ Tiempo de reacción (s) } & \multicolumn{2}{|c|}{ Hombres } & \multicolumn{2}{c|}{ Mujeres } & \multirow{2}{*}{} \\
\hline & Mediana & Pc: 25-75 & Mediana & Pc: $25-75$ & p \\
\hline Lámina 1 & 3 & 2 a 4 & 2 & 1 a 4 & 0,28 \\
\hline Lámina 2 & 5 & 3 a 9 & 5 & 2 a 8 & 0,6 \\
\hline Lámina 4 & 5 & 3 a 10 & 4 & 3 a 6 & 0,34 \\
\hline Lámina 10 & 8 & 4 a 11 & 6 & 3 a 13 & 0,6 \\
\hline Lámina 13 & 9 & 4 a 14 & 6 & 4 a 11 & 0,24 \\
\hline Lámina 14 & 4 & 3 a 7 & 4 & 2 a 6 & 0,42 \\
\hline
\end{tabular}

La lámina con el mayor tiempo total para los hombres es la lámina $8 \mathrm{VH}$-"La intervención quirúrgica”- (media de 168,63 segundos) y para las mujeres es la lámina 2 - "La estudiante en el campo"- (media de 174,74 segundos). La lámina con el menor tiempo total para los hombres es la lámina 1 -"El chico y el violín”- (media de 87,11 segundos) y para las mujeres es la lámina $3 \mathrm{NM}$-"La joven en la puerta"- (media de 80,45 segundos). En las tablas de Rosenzweig (6), los hombres también emplearon mucho menos tiempo en la lámina 1 -"El chico y el violín"-; en cambio, la lámina 13 HM -"Mujer en la cama"- les ocupó el tiempo total más largo. Las mujeres también dieron el tiempo mínimo en la lámina $3 \mathrm{NM}$-"La joven en la puerta"- y el máximo en la 9 NM, "Dos mujeres en la playa".

TABLA 6

Tiempo total

\begin{tabular}{|l|c|c|c|c|c|}
\hline \multirow{2}{*}{ Tiempo total (s) } & \multicolumn{2}{|c|}{ Hombres } & \multicolumn{2}{c|}{ Mujeres } & \\
\hline & Mediana & Pc: 25- 75 & Mediana & Pc: 25- 75 & p \\
\hline Lámina 1 & 83 & 59 a 113 & 79 & 57 a 90 & 0,61 \\
\hline Lámina 2 & 144 & 119 a 192 & 155 & 100 a 205 & 0,8 \\
\hline Lámina 4 & 128 & 115 a 154 & 119 & 94 a 145 & 0,22 \\
\hline Lámina 10 & 84 & 69 a 107 & 77 & 60 a 142 & 0,9 \\
\hline
\end{tabular}




\begin{tabular}{|l|c|c|c|c|c|}
\hline Tiempo total (s) & \multicolumn{2}{|c|}{ Hombres } & \multicolumn{2}{c|}{ Mujeres } & \\
\hline & Mediana & Pc: 25- 75 & Mediana & Pc: 25- 75 & p \\
\hline Lámina 13 & 157 & 103 a 179 & 144 & 113 a 175 & 0,75 \\
\hline Lámina 14 & 90 & 70 a 121 & 85 & 57 a 133 & 0,52 \\
\hline
\end{tabular}

La lámina que ha generado mayor número de palabras para los hombres es la lámina $8 \mathrm{VH}$-"La intervención quirúrgica"- (media de 199,52 palabras) y para las mujeres es la lámina 2 -"La estudiante en el campo"- (media de 163,22 palabras). La lámina que ha generado menor número de palabras para los hombres es la lámina 1 - "El niño y el violín"- (media de 94,85 palabras), y para las mujeres es la lámina 10 -"El abrazo"- (media de 72,91 palabras). Rosenzweig escribió sobre la lámina 1 que, o bien a esta altura del examen el examinado no está todavía "en forma", o que esta lámina es menos incentivadora que las restantes. Murray (27) informó de que 300 palabras constituyen la duración estándar para las historias de adultos. McClelland y colaboradores (28) consideraron 100 palabras como el recuento mínimo suficiente, y Skolnick (29) logró establecer asociaciones con historias todavía más cortas.

Como muestra la Tabla 7, la comparación entre sexos únicamente arroja resultados estadísticamente significativos en cuanto al número de palabras. Este dato es significativamente menor en el caso de las mujeres durante la lámina 14 -"El hombre en la ventana”-. El número de palabras de las láminas 1 -"El niño y el violín”- y 4 - "Mujer que retiene al hombre"- están próximos a la significación estadística, también a favor de los hombres.

TABLA 7

Número de palabras

\begin{tabular}{|l|c|c|c|c|c|}
\hline Número de palabras & \multicolumn{2}{|c|}{ Hombres } & \multicolumn{2}{c|}{ Mujeres } & \multirow{2}{*}{} \\
\hline & Mediana & Pc: $25-75$ & Mediana & Pc: $25-75$ & p \\
\hline Lámina 1 & 91 & 66 a 108 & 75 & 42 a 88 & 0,07 \\
\hline Lámina 2 & 186 & 102 a 243 & 147 & 73 a 232 & 0,26 \\
\hline Lámina 4 & 144 & 86 a 193 & 97 & 59 a 150 & 0,07 \\
\hline Lámina 10 & 97 & 51 a 126 & 61 & 40 a 90 & 0,1 \\
\hline Lámina 13 & 147 & 95 a 230 & 117 & 74 a 155 & 0,17 \\
\hline Lámina 14 & 93 & 76 a 136 & 69 & 45 a 102 & $\mathbf{0 , 0 4 4}$ \\
\hline
\end{tabular}


Pasemos al contenido de las láminas (las tablas detalladas con todas las variables estudiadas pueden consultarse en el Anexo).

En la lámina 1 -"El chico y el violín"-, los sujetos narran la historia de un niño (descrito más frecuentemente como triste en el caso de las mujeres, e interesado en la música en el caso de los hombres; ambos datos próximos a la significación estadística), pensando si podrá tocar el violín (si tendrá la capacidad suficiente para hacerlo) y cuyo desenlace más frecuente es que no pueda (que no tenga la capacidad suficiente). Los pacientes de nuestro estudio, además, suelen omitir la hoja de música o papel. En la tabla de Rosenzweig (6), la historia más frecuente es la de un nińo, hijo de padres ambiciosos que quieren que estudie música, mirando el violín, que puede resistirse o no a practicar. En la tabla de Ávila Espada (7), la historia más frecuente es la de un niño, hijo de padres que quieren que estudie música, pensando en el éxito futuro.

Esta lámina le abre la puerta al reconocimiento y proyección de emociones en esquizofrenia. Ya hemos comentado cómo los examinados connotan al niño como triste y con sentimientos de incapacidad que pueden jugar un papel en el hecho de que finalmente no pueda tocar el violín. De aquí en adelante, encontraremos que al menos 17 de las 31 figuras analizadas son descritas, en diferentes órdenes de frecuencia, como "tristes", siendo uno de los sentimientos más frecuentemente narrados en esta población. Este sentimiento puede estar en relación con la muerte, como veremos a continuación, que es causa o desenlace de varias escenas dramáticas en esta población.

Algunos pacientes, pese a su edad, han adaptado culturalmente la lámina a los tiempos actuales y no dudan en identificar un ordenador en lugar del violín o en explicar que el niño sostiene un teléfono móvil entre su mano y su oreja.

En la lámina 2 -"La estudiante en el campo"-, los sujetos narran la historia de dos figuras femeninas, una estudiante (descrita más frecuentemente por las mujeres como joven, con significación estadística) y otra mujer, que están esperando a que la figura en el fondo termine de trabajar. La figura del fondo es un hombre (descrito más frecuentemente por los hombres como marido y padre de las dos figuras femeninas, y llamando la atención sobre su desnudez. Resulta estadísticamente significativo que los hombres traten de aclarar las relaciones de parentesco que se dan entre las figuras de la lámina), agricultor, que está trabajando un campo (descrito más frecuentemente por los hombres como con unos surcos, próximo a la significación estadística) junto a un caballo que le puede dar problemas. En la tabla de Rosenzweig (6), la historia más frecuente es la de una estudiante adolescente, hija de las otras dos figuras, que quiere abandonar la granja. En la tabla de Ávila Espada (7), la historia más frecuente es la de una estudiante joven, hija de las otras dos figuras o vecina del lugar, que quiere abandonar la tierra y el campo. 
Para muchos pacientes que narran problemas con el caballo, esta percepción viene originada por la supuesta discrepancia entre la dirección a la que apunta el hombre con su mano y la dirección hacia la cual dirige la testa el animal.

En la lámina $3 \mathrm{VH}$-"Reclinado/a en el diván”-, los hombres narran la historia de una muchacha enferma mental o pobre que descansa o duerme en un banco, llorando por un problema amoroso. Los pacientes de nuestro estudio, además, suelen omitir el objeto en el suelo. En la tabla de Rosenzweig (6), la historia más frecuente es la de una figura femenina, que podría ser una nińa, criminal o prisionera, que descansa o duerme junto a un objeto en el suelo, llorando por una infancia desgraciada o una tragedia matrimonial o romántica. En la tabla de Ávila Espada (7), la historia más frecuente es la de una figura femenina, que puede ser nińa o joven, que llorando tristemente lamenta su situación. Esta situación es consecuencia de una tragedia o problema matrimonial, amoroso o sexual.

En la lámina $3 \mathrm{NM}$-"La joven en la puerta"-, las mujeres narran la historia de una muchacha llorando junto a una puerta por la muerte de algún ser querido o por una desgracia familiar. En la tabla de Rosenzweig (6), la historia más frecuente es la de una mujer joven, en una habitación con una puerta a través de la cual se ve otra habitación, disgustada o apenada por la muerte de algún ser querido o por un fracaso matrimonial o romántico. En la tabla de Ávila Espada (7), la historia más frecuente es la de una mujer joven llorando apenada por problemas matrimoniales o románticos.

En la lámina 4 -"Mujer que retiene al hombre"-, los sujetos narran la historia de un hombre y una mujer, ambos actores de cine, que son pareja. El hombre se muestra desinteresado por la mujer, y la mujer se muestra interesada por el hombre (descrita más frecuentemente por los hombres como consolando o calmando al hombre, con significación estadística). Al fondo se aprecia una figura humana femenina. Algunos hombres, además, sitúan la acción en un bar, cabaret, teatro, casa de citas, burdel o prostíbulo y ven la figura humana femenina al fondo desnuda o semidesnuda. La pareja no se entiende o riñen, siendo el desenlace más frecuente que acaben separados. En la tabla de Rosenzweig (6), la historia más frecuente es la de un hombre y una mujer jóvenes, que son marido y mujer. Al fondo se ve un cuadro. Ambos están angustiados y la mujer trata de evitar que el hombre la abandone. En la tabla de Ávila Espada (7), la historia más frecuente es la de un hombre irritado y una mujer seductora, que son marido y mujer, donde la mujer trata de evitar que el hombre la abandone.

Un $24 \%$ de los pacientes de nuestro estudio refieren que se trata de una película (Ávila Espada señala cómo un 5\% de los pacientes de su estudio relatan que es una escena tipo films "H. Bogart", 7). El hecho de que para muchos pacientes esta lámina se trate del fotograma de una película impregnará las descripciones de las restantes láminas del test. No será raro que a partir de este momento los examinados 
se refieran a las figuras de las próximas láminas como "este actor..." o "esta actriz..." a la hora de describirlas. No consideramos que sea la percepción de una misma persona en distintas láminas.

Muchos autores han recalcado hasta qué punto la integridad de la función mnésica es un factor limitante para ofrecer historias con un material rico. En esta lámina, llama la atención el reconocimiento que algunos examinados hacen de la supuesta actriz, identificándola como Bette Davis, Judy Garland, Susan How o Hayward, Ava Gardner, Sophia Loren o Angelina Jolie.

En la lámina $6 \mathrm{VH}$-"El hijo que se va"-, los hombres narran la historia de una madre vieja y apenada junto a una ventana con su hijo, también triste, tras la muerte de un familiar, que puede ser el marido y padre, respectivamente. En la tabla de Rosenzweig (6), la historia más frecuente es la de una mujer vieja, madre de un hombre joven, en la que ambos están angustiados o disgustados, y los negocios u otro deber exigen que el hijo abandone el hogar. En la tabla de Ávila Espada (7), un hombre joven y su anciana madre están tristes por haber recibido una mala noticia: la muerte o grave enfermedad del marido y padre de las dos figuras.

En la lámina $7 \mathrm{VH}$-"Padre e hijo"-, los hombres narran la historia de un hombre mayor, padre de la otra figura, que está aconsejando al hombre más joven. En la tabla de Rosenzweig (6), la historia más frecuente es la de un hombre mayor, de carrera, padre del hombre más joven, al que aconseja o consuela. En la tabla de Ávila Espada (7), un hombre adulto, padre del hombre joven, le está aconsejando o consolando. El hombre joven está triste o preocupado por problemas.

Para algunos examinados, padre e hijo no se entienden o riñen. Al igual que ocurría en la lámina 2 entre el agricultor y el caballo (y volverá a ocurrir en otras láminas como la $7 \mathrm{NM}$ o la $12 \mathrm{M}$ ), el hecho de que dos figuras no se miren la una a la otra o no miren en la misma dirección puede ser fuente de conflicto, tanto para hombres como para mujeres.

En la lámina 7 NM -"Niña y muñeca"-, las mujeres narran la historia de una madre leyéndole a su niña, que mira para otro lado inatenta o desinteresada de lo que le dice su madre, y está triste. Las figuras no se entienden o riñen. En la tabla de Rosenzweig (6), la historia más frecuente es la de una mujer, madre de la otra figura, que está enseñando o ilustrando a la niña. En la tabla de Ávila Espada (7), una niña está distraída o fastidiada mientras su madre, una mujer adulta, le lee con intención de distraerla o enseñarle.

En la lámina $8 \mathrm{VH}$-"La intervención quirúrgica"-, los hombres narran la historia de un niño (hasta adolescente) en primer plano, hijo en un $22,2 \%$ de las respuestas de la figura masculina echada, que ha sido disparada, mientras dos doctores le atienden. En la tabla de Rosenzweig (6), la historia más frecuente es la de un niño (hasta adolescente) que está imaginando la escena en el fondo, en la que un doctor y 
su ayudante se hallan con un paciente. Esta escena estimula al muchacho a hacerse doctor. En la tabla de Ávila Espada (7), un niño o adolescente, que puede ser el hijo de la figura tumbada (un 8,5\% respondió a este parentesco), está triste o angustiado, imaginando la escena del fondo. La figura tumbada ha sido víctima de agresión o combate y dos médicos le están operando.

En la lámina 9 NM - "Dos mujeres en la playa”-, las mujeres narran la historia de dos mujeres que van corriendo o con prisa ambas, que bien pueden ser madre e hija o amigas, y mantienen un conflicto. En la tabla de Rosenzweig (6), la historia más frecuente es la de dos mujeres jóvenes que son rivales o hermanas y mantienen un conflicto. La figura de la derecha, arriba o en primer plano se está escondiendo de la otra mujer, que va corriendo y se encuentra emocionalmente turbada. En la tabla de Ávila Espada (7), la historia más frecuente es la de una mujer joven que está espiando a otra mujer joven que va corriendo. Las mujeres pueden ser amigas o hermanas, y mantienen un conflicto.

En la lámina 10 -"El abrazo”-, existen problemas de identificación del sexo -sobre todo de la figura a la derecha (un $63 \%$ de los hombres y un 52,2 \% de las mujeres la consideran femenina)-, por lo que se intercalan las historias de dos figuras que pueden ser pareja, padre/madre e hijo/hija, o amigos. Se quieren y están encontrándose. En la tabla de Rosenzweig (6), la historia más frecuente es la de una figura masculina a la izquierda y una figura femenina a la derecha que se abrazan enamorados. En la tabla de Ávila Espada (7), también parecen existir problemas, aunque de menor magnitud, para identificar el sexo de la figura de la derecha (un $71,1 \%$ de los hombres y un $63,8 \%$ de las mujeres la consideran femenina). En su tabla se cuenta la historia de un hombre y una mujer que son marido y mujer o pareja, enamorados, abrazándose y/o bailando, que proseguirán juntos su vida feliz.

Muchos examinados comienzan la narración de esta historia, que abortan y reinician cuando se presentan dudas sobre el sexo de las figuras y su combinación. La narración puede bloquearse y entorpecerse, presentando y descartando con torpeza distintas alternativas.

En la lámina $12 \mathrm{M}$ - "La celestina”-, las mujeres narran la historia de una mujer joven asustada que no quiere mirar a la otra mujer mayor, bruja, que es su madre y trata de perjudicarla. La mujer mayor, sin embargo, está feliz. En la tabla de Rosenzweig (6), la historia más frecuente es la de una mujer joven, hija o nieta de la mujer vieja, que está atenta, centrada en sí misma y angustiada. La mujer vieja es malvada o astuta, y está influyendo adversamente sobre la más joven.

En la lámina 13 HM - "Mujer en la cama”-, los sujetos narran la historia de una mujer desnuda y muerta (descrita más frecuentemente por las mujeres como enferma), que es la pareja de un hombre que se tapa los ojos mientras llora (descrito más frecuentemente por los hombres como de pie y preocupado, con significación 
estadística). El desenlace más frecuente en el caso de los hombres es que el hombre ha matado a la mujer, y en el caso de las mujeres es que la mujer ha muerto por enfermedad. En la tabla de Rosenzweig (6), la historia más frecuente es la de un hombre joven, compańero sexual ilícito de la mujer, que está lleno de remordimiento. La mujer, también joven, está muerta (era más frecuente que las mujeres la describieran como asesinada) y el hombre ha tenido o está meditando la unión con la mujer.

En la lámina 14 -"Hombre en la ventana"-, los sujetos narran la historia de un hombre mirando por una ventana en una habitación oscura (en la que también existe una luz o claridad descrita más frecuentemente por los hombres, con significación estadística) de la que quiere escapar. En la tabla de Rosenzweig (6), la historia más frecuente es la de un hombre adolescente (hasta los veintitantos) que está contemplando el cielo, pensando o sońando con su futuro. En la tabla de Ávila Espada (7), la historia más frecuente es la de un hombre joven contemplando el cielo por la noche, para relajarse y descansar. Tras mirar hacia fuera, volverá a su rutina.

En la lámina $18 \mathrm{VH}$-"Atacado desde atrás"-, los hombres narran la historia de un hombre enfermo al que las manos de otra figura masculina que es amigo o compañero suyo están ayudando a sostenerse. En la tabla de Rosenzweig (6), la historia más frecuente es la de un hombre alcoholizado y angustiado, en la que se relatan un número confuso de manos, que pueden ser de la policía, que le están atacando realmente, en sueños o arrestándole por un acto criminal. En la tabla de Ávila Espada (7), un hombre, que puede estar inconsciente o angustiado. Unas manos masculinas pueden estar atacándole, arrestándole o ayudándole. El hombre está borracho o se emborrachará.

En la lámina $18 \mathrm{NM}$-"Mujer que estrangula"-, las mujeres narran la historia de una mujer mayor (figura a la izquierda) que está enferma, mientras su hija, que es una niña, la sujeta, llorando y triste. En la tabla de Rosenzweig (6), la historia más frecuente es la de una mujer angustiada (figura a la derecha) que está sosteniendo a otra figura en la que los sujetos presentan problemas de identificación del sexo, que es joven y está enfermo/a. Pueden ser marido y mujer o una madre que está dando ayuda o consuelo a su hijo/a en enfermedad, daño o susto. En la tabla de Ávila Espada (7), la historia más frecuente es la de una mujer adulta (figura de la derecha), madre de la otra figura, que puede estar auxiliando o -en menor medida- estrangulando a la otra mujer adulta, que está enferma.

\section{Discusión}

Este estudio propone varias historias convencionales preferidas entre la población institucionalizada con trastorno mental grave y prolongado.

Se entiende que aquellas historias que se asemejen más a las descritas por Rosenzweig y Ávila Espada serán las menos interesantes para discriminar a la población 
con trastorno mental grave y prolongado. Estas historias son las relativas a las láminas $3 \mathrm{VH}$ "Reclinado en el diván", 7 VH "Padre e hijo", 7 NM "Niña y muñeca", 9 NM "Dos mujeres en la playa”, 10 "El Abrazo" y 12 "La celestina”. Aunque estas láminas puedan resultar menos interesantes de cara al contenido de las historias, no desaconsejamos su aplicación por otros motivos. Por ejemplo, para comprobar omisiones en la lámina $3 \mathrm{VH}$, observar la tendencia a concluir que dos figuras rińen por el hecho de que no estén mirando hacia el mismo lado en las láminas 7 VH y 7 NM, comprobar si existe un tiempo de reacción alto con respecto al resto de la batería en la lámina 9 NM o los problemas de identificación del sexo de la figura de la derecha en la lámina 10, así como la tendencia de las figuras a encontrarse tristes y a la inactividad. La aplicación de las láminas restantes de la batería propuesta la consideramos, a todas luces, útil para la evaluación del paciente con trastorno mental grave y prolongado. Ávila Espada ya incidió en la posibilidad de eliminación de las láminas menos productivas y el reaprovechamiento de láminas diseñadas para propósitos de investigación (7), útiles por su poder discriminatorio para ciertos temas y para las que haya un cuerpo de datos normativos suficiente.

Los pacientes de nuestro estudio, además, describen el mobiliario presente y la vestimenta de las figuras en numerosas láminas, lo que no aparece en las tablas de Rosenzweig (6). Otros se empeñan en datar a qué siglo corresponden las historias. Desconocemos si Rosenzweig no dio importancia a esos detalles, o tal vez se encontraban presentes en un número menor al $20 \%$ de su muestra y por ello no fueron tabulados.

Se acepta que la tabulación es la forma más larga, laboriosa y dispendiosa de establecer un marco referencial para la elaboración del TAT, pero compensa, puesto que confiere una mayor seguridad y precisión a la interpretación, además de evitar desatenciones u omisiones en el análisis. Sin embargo, lo engorroso de algunos sistemas que sirven de guía (como el ideado por Tomkins (30), que recae en una clasificación frase por frase en la que aplica 37 variables) ha desalentado al lector de TAT a lo largo de la historia, extendiendo la desilusión al TAT mismo (31).

Las mujeres de nuestro estudio tienden a emplear un menor número de palabras para desarrollar el contenido de sus historias, estadísticamente significativo en el caso de la lámina 14 - "El hombre en la ventana”-. Este dato podría corresponderse con una mayor pobreza del discurso con respecto a los hombres. Sin embargo, a la hora de valorar estos hallazgos debe advertirse que las mujeres del estudio cuentan con un mayor número de años de hospitalización respecto a los hombres, una diferencia que resultó estadísticamente significativa.

No solo debemos tener en cuenta el contenido de las historias para esta población, sino que también debemos llamar la atención sobre el repertorio del apartado "Problemas y soluciones" que han facilitado los sujetos, por ser cuantitativamente inferior a los 41 problemas y soluciones que hallara Rosenzweig para la misma serie 
de láminas. Esta aparente pobreza imaginativa concordaría con lo expuesto por la psicóloga Rosa Sanguino y colaboradores en un estudio longitudinal de variables cognitivas en mujeres con esquizofrenia durante un seguimiento a 31 ańos en el mismo centro sociosanitario del presente estudio, en el que se desvela que este tipo de sujetos tienen un bajo nivel cognitivo (32). En el citado estudio también se demostró un decalaje de variables cognitivas en etapas finales de la vida, lo que complica la tarea de diferenciar si la pobreza en el repertorio de historias del TAT en este tipo de sujetos puede deberse al bajo nivel cognitivo de base o a un deterioro superpuesto asociado a la edad. Para dificultar más la tarea, debemos tener en cuenta que la media de edad de los sujetos de nuestro estudio es considerablemente superior a las edades de los sujetos de los estudios de Rosenzweig y de Ávila Espada. Otros factores sobreañadidos y combinables entre sí que podrían estar influyendo sobre la pobreza imaginativa son: el nivel educativo bajo, el uso de psicofármacos, la cronicidad o el predominio de sintomatología negativa.

No es extraño que los sujetos de nuestro estudio se limiten a describir la lámina dejando la historia sin resolución, contestando que las figuras "se quedan asi" incluso cuando se les anima a inventar un desenlace.

Otra limitación la encontramos a la hora de exponer el contenido de las historias, aunque solo lo hagamos descriptivamente, en relación a los hallazgos de Rosenzweig (6). Aunque esta comparación nos parezca pertinente, no podemos olvidar que se trata de un estudio muy antiguo y no es específico de nuestro país por tratarse de población norteamericana. Sin embargo, Ávila Espada señala que las normas españolas no son para nada diferentes de las estadounidenses o las francesas, a pesar de algunos elementos culturales y situacionales específicos (33).

Ahora que hemos tratado de mostrar los resultados de la forma más rigurosa posible, debemos ser honestos exponiendo, a nuestro entender, la filosofía del TAT. Vivimos en la era de la medicina basada en la evidencia, abanderada por las pruebas científicas provenientes de la investigación científica metodológicamente correcta para la toma de decisiones objetiva. Este hecho suele chocar con la naturaleza del TAT, cuya elaboración (aun dejando fuera la interpretación) está influenciada por la subjetividad del examinador que la haya llevado a cabo. En nuestra opinión, esta subjetividad no desvaloriza la calidad del material obtenido. De hecho, dado que el propósito del TAT no es la toma de decisiones unilaterales basadas en sus resultados, consideramos que incorporar las experiencias del examinador se erige como una herramienta de uso perentorio para su completo aprovechamiento. Dichas experiencias forman parte inherente del producto final, y están incluidas en el presente apartado de discusión.

Los test proyectivos, como el TAT y el Rorschach, no solo replican o confirman hallazgos obtenibles mediante exámenes más rápidos, más fáciles de obtener y 
menos costosos de realizar (como los cuestionarios autoinformados), sino que pueden hacer aportaciones únicas en la planificación, monitorización y evaluación de resultados en psicoterapia. Los estudios sobre su utilidad en estas cuestiones deberían ser diseñados para iluminar la cantidad de información útil que proveen que se hubiera perdido sin ellos (34). Las técnicas utilizadas para evaluar las historias han sido criticadas metodológicamente pese a la sustancial literatura acumulada al respecto. El siguiente paso consiste en ir más allá de las reglas obsoletas relativas a los test clásicos y crear una literatura moderna para el TAT que apoye el uso más completo de la riqueza que ofrece (35).

Como ventajas del estudio, cabe señalar que se ha realizado sobre una amplia muestra de población difícilmente replicable desde la reforma psiquiátrica en España, recopilando historias en pacientes con esquizofrenia de muchos años de evolución. Además, se realizaron 187 contrastes de hipótesis. Según Streiner, muestras pequeñas pueden malgastar la energía de los investigadores en esfuerzos de recopilación de datos, la mayoría de los cuales están destinados a ser infructuosos (36). La mayoría de los estudios sobre el TAT, además, se han efectuado en población más joven.

Como desventajas del estudio, podemos citar que no ha sido un estudio ciego y que ha estado influenciado por la configuración de normas aperceptivas que estableciera Rosenzweig el siglo pasado. El propio Rosenzweig presentó problemas semejantes a la hora de tabular (por ejemplo, un relato que incluía asesinato lo clasificó como asesinato o como hostilidad; cuando una historia contenía más de una caracterización, tabuló sólo el aspecto dominante; etc.) y advirtió que "algunas arbitrariedades durante la clasificación de los datos fueron inevitables". Además, la selección y extracción de datos específicos procedentes de las historias para su conversión a formato de tablas es un proceso que varía según la personal orientación escolástica del examinador, destreza, conocimientos, propósito de la aplicación y tiempo disponible, y que no ha estado exento de dificultades (por ejemplo, en historias de muy pocas palabras, el examinador debía inferir el sexo de las figuras a través del género gramatical empleado por el examinado para referirse a ellas). En estudios futuros, podrían establecerse grupos de examinadores para la toma de decisiones en materia de análisis en los que se discuta cómo tabular, adaptando las normas aperceptivas a la idiosincrasia del sujeto narrador y disminuyendo el impacto dependiente de la personalidad de un único examinador. No se empleó ningún sistema manualizado para puntuar las historias del TAT por beneficiarse estos sistemas de las historias largas, sin embargo, la literatura preexistente ha mostrado que las historias suministradas por pacientes con esquizofrenia tienden a ser cortas. Queremos resaltar que aunque los sistemas manualizados para puntuar el TAT facilitan la investigación, el entrenamiento y la aplicación clínica de ninguna manera dan cuenta de toda la riqueza de la información disponible cuando una persona cuenta una historia (37). 
Acerca de los problemas metodológicos que plantea la investigación con test proyectivos, nos gustaría añadir algunas consideraciones. Se habla de la dificultad de llegar a generalizaciones válidas a partir de los instrumentos proyectivos, o bien se remarca la falta de estandarizaciones y normas. Algunos estudios piensan que es factible utilizar métodos proyectivos, como también utilizar instrumentos de manera creativa, aplicando una relectura de uno ya vigente y validado (38). Sharon Rae Jenkins defiende que las críticas a las técnicas de apercepción temática omiten estudios que evidencian la validez de la prueba según su uso, malinterpretando el propósito de los estudios. En respuesta a lo que considera ataques al TAT, replica enfatizando que el examen responde a preguntas clínicas que son demasiado complejas e interactivas como para ser empíricamente probadas con la metodología de que disponemos actualmente, y recalca que herramientas metodológicamente imperfectas pueden ser suficientemente valiosas respondiendo a tareas clínicas específicas y para comprender pautas de comportamiento recurrentes. Esta autora sostiene que la dificultad para reportar hallazgos en materia de investigación alimentada por estas críticas trunca estrategias económicas relacionadas con el entrenamiento y la aplicación de estos test. En su opinión, las innovaciones que necesitan las técnicas aperceptivas no son siempre científicas y aboga por la comunicación entre investigadores interesados en la materia para que compartan sus esfuerzos y denuncien las dificultades encontradas (39).

Algunos autores, como Rapaport, no consideran necesario disponer de tablas, pues creen que es mejor que el examinador no se vea limitado por estas enumeraciones o forzado a decidir de antemano qué debe buscar. En cualquier caso, no parece discutible su valor didáctico y de guía para el interesado en TAT, que debe recordar que el ajuste a las normas aperceptivas para un grupo específico debe determinarse mediante la consideración de la totalidad del repertorio de historias dentro de ese mismo grupo. Estas determinaciones se sirven de la comparación entre grupos y la intuición adquirida por el examinador en base a su experiencia y conocimientos procedentes de su formación y otras fuentes. Los resultados del presente estudio no pretenden ser empleados para dirigir paso a paso la inspección, más bien se trata de un marco referencial más que los examinadores pueden incorporar y utilizar libre y espontáneamente en sus análisis.

\section{BiBLIOGRAFÍA}

(1) Morgan WG. Origin and history of the earliest thematic apperception test pictures. J Pers Assess. 2002;79(2):422-445.

(2) Bernstein J. Introducción al TAT. Test de apercepción temática (TAT). Manual para la aplicación. Buenos Aires: Editorial Paidós, 1979. 
(3) Castilla del Pino C. Obras completas. Volumen VII. Córdoba: Editorial Fundación Castilla del Pino, Universidad de Córdoba, 1980.

(4) Castilla del Pino C. Teoría de los sentimientos. Barcelona: Tusquets Editores, 2009.

(5) França E. Thematic Apperception Test: Algunas situaçoes tipicas dentro da amostra de adultos examinados no ISOP. Arquivos Brasileiros de Psicotécnica 1953; 1 y 4.

(6) Rosenzweig S. Apperceptive norms for the Thematic Apperception Test: II. An empirical investigation. J. Personality 1949;17:475-503.

(7) Ávila Espada A. El test de apercepción temática de H. M. Murray en la población española: estudio normativo y análisis para una adaptación. Memoria para optar al grado de doctor. Madrid: Universidad Complutense de Madrid, 2015.

(8) Jones J. Transactional style deviance in families of disturbed adolescents. Tesis doctoral. Los Angeles, CA: University of California at Los Angeles (UCLA), 1974.

(9) Manschreck TC, Merrill AM, Jabbar G, Chun J, Delisi LE. Frequency of normative word associations in the speech of individuals at familial high-risk for schizophrenia. Schizophr Res. 2012;140(1-3):99-103.

(10) Jones JE. Patterns of transactional style deviance in the TAT's of parents of schizophrenics. Fam Process. 1977;16(3):327-37.

(11) Singer MT, Wynne LC. Principles for scoring communication defects and deviances in parents of schizophrenics: Rorschach and TAT scoring manuals. Psychiatry. 1966; 29(3):260-88.

(12) Doane JA, Miklowitz DJ, Flores, R, Karno, M, Strachan AM, Jenkins JH. Parental communication deviance and schizophrenia: a cross-cultural comparison of Mexican-and Anglo-Americans. J Abnorm Psychol. 1989; 98(4):487-490.

(13) Meyer RG, Karon BP. The schizophrenogenic mother concept and the TAT. Psychiatry. 1967; 30(2):173-9.

(14) Escartín Alcubierre LC. Rasgos de personalidad en las madres de pacientes esquizofrénicos. Tesis doctoral. Zaragoza: Universidad de Zaragoza, 1988.

(15) Waszkiewicz J, Wciórka J, Anczewska M, Chrostek A, Switaj P. Language disorders and cognitive functions in persons with schizophrenic disorders. Psychiatr Pol. 2012; 46(4):553-70.

(16) Lysaker PH, Daroyanni P, Ringer JM, Beattie NL, Strasburger AM, Davis LW. Associations of awareness of illness in schizophrenia spectrum disorder with social cognition and cognitive perceptual organization. J Nerv Ment Dis. 2007;196(7):618-21.

(17) Kenchadze VH, Chkoniia ED. Clinical features of cannabis psychosis in schizophrenia patients. Georgian Med News. 2006;(137):55-8.

(18) Cabal Bravo JC, Bobes García J, Vázquez Fernández A, González-Quirós Corujo P, Bousoño García M, García Prieto A, et al. Thematic Apperception Test: psychodiagnosis in heroin-dependent patients. Actas Luso Esp Neurol Psiquiatr Cienc Afines. 1990; 18(1):1-6.

(19) Fowler JC, Ackerman SJ, Speanburg S, Bailey A, Blagys M, Conklin AC. Personality and symptom change in treatment-refractory inpatients: evaluation of the phase model of change using Rorschach, TAT, and DSM-IV Axis V. J Pers Assess. 2004;83(3):306-322.

(20) Litinsky AM, Haslam N. Dichotomous thinking as a sign of suicide risk on the TAT. J Pers. Assess.1998;71(3):368-378. 
(21) Bouvet C, Cleach C. Early dropout patients in psychiatric psychosocial rehabilitation treatment and their bindings with relational skills, object relation and intensity of psychopathology. Encephale 2011;37(Suppl 1):S19-26.

(22) Stein MB, Slavin-Mulford J, Sinclair SJ, Siefert CJ, Blais MA. Exploring the construct validity of the social cognition and object relations scale in a clinical sample. $\mathrm{J}$ Pers. Assess. 2012; 94(5): 533-40.

(23) Limosani I, D’Agostino A, Manzona ML, Scarone S. Bizarreness in dream reports and waking fantasies of psychotic schizophrenic and manic patients: Empirical evidences and theoretical consequences. Psychiatry Res. 2011;189(2): 195-199.

(24) Weisskopf EA. A transcendence index as a proposed measure in the TAT. J Psychol. 1950; 29: 379-390.

(25) Luque Luque R. Esquizofrenia aguda: Análisis del lenguaje (Tesis doctoral). Córdoba: Universidad de Córdoba, 2008.

(26) Moritz S, Woodward TS. Plausibility judgment in schizophrenic patients: evidence for a liberal acceptance bias. Ger J Psychiatry 2004; 7(4): 66-74.

(27) Murray H. Manual for the Thematic Apperception Test. Cambridge, MA: Harvard University Press, 1943.

(28) McClelland DC, Atkinson JW, Clark RA, Lowell EL. The achievement motive. New York: Irvington Publishers Inc., 1953.

(29) Skolnick A. Motivational imagery and behavior over twenty years. Journal of Consulting Psychology 1966; 30: 463-478.

(30) Tomkins SS, Tomkins EJ. The Thematic Apperception Test. The theory and technique on interpretation. New York: Ed. Grune \& Stratton, 1948.

(31) Pichot P. Les tests mentaux en psychiatrie. París: Presses Universitaires de France, $1949 ;$ p. 195.

(32) Sanguino-Andrés R, López-Villalobos JA, González-Pablos E, Guarido-Rivera V, González-Sanguino C, López-Sánchez MV, et al. Longitudinal study of cognitive variables in women with schizophrenia: 31-year follow-up study. Span J Psychol. 2015; 18:E92.

(33) Ávila-Espada A (2000). Objective scoring for the TAT. En: Dana RH (Ed.) Handbook of cross-cultural and multicultural personality assessment. Mahwah, NJ: Lawrence Erlbaum Associate Publishers, 2000; pp. 465-480.

(34) Weiner IB. Monitoring psychotherapy with performance-based measures of personality functioning. J Pers Assess. 2004; 83(3): 323-331.

(35) Jenkins SR. Not your same old story: new rules for thematic apperceptive techniques (TATs). J Pers Assess. 2017; 99(3): 238-253.

(36) Streiner DL. Sample size in clinical research: When is enough? J Pers Assess. 2006; 87: 259-260.

(37) Karon BP. The clinical interpretation of the Thematic Apperception Test, Rorschach, and other clinical data: a reexamination of statistical versus clinical prediction. Prof Psychol Res Pract. 2000; 31: 230-233.

(38) Sneiderman S. Consideraciones acerca de la confiabilidad y validez en las técnicas proyectivas. Subjetividad y procesos cognitivos 2011; 15(2): 93-110.

(39) Jenkins SR. The narrative arc of TATs: introduction to the JPA special section on thematic apperceptive techniques. J Pers Assess. 2017; 99(3): 225-237. 


\begin{tabular}{|c|c|c|c|c|}
\hline \multicolumn{5}{|l|}{ Lámina 1 "El chico y el violín" } \\
\hline I. FIGURAS & Total & Hombres & Mujeres & $\mathbf{p}$ \\
\hline \multicolumn{5}{|l|}{ A. Figura sola } \\
\hline \multicolumn{5}{|l|}{ 1. Sexo } \\
\hline Masculino & $100 \%$ & $100 \%$ & $100 \%$ & \\
\hline \multicolumn{5}{|l|}{ 2. Edad } \\
\hline Nińo, chico o muchacho & $96 \%$ & $96,3 \%$ & $95,7 \%$ & 1 \\
\hline \multicolumn{5}{|l|}{ 3. Identidad } \\
\hline \multicolumn{5}{|l|}{ Sin resultados } \\
\hline \multicolumn{5}{|l|}{ 4. Otras caracterizaciones } \\
\hline Pensativo o pensando & $46 \%$ & $40,7 \%$ & $52,2 \%$ & 0,57 \\
\hline Mirando al violín & $28 \%$ & $33,3 \%$ & $21,7 \%$ & 0,53 \\
\hline $\begin{array}{l}\text { Dormido, como dormido, } \\
\text { durmiéndose, medio dormido o } \\
\text { casi dormido }\end{array}$ & $26 \%$ & $25,9 \%$ & $26,1 \%$ & 1 \\
\hline Estudiando & $24 \%$ & $22,2 \%$ & $26,1 \%$ & 1 \\
\hline $\begin{array}{l}\text { Ojos cerrados, medio cerrados } \\
\text { o casi cerrados }\end{array}$ & $20 \%$ & $25,9 \%$ & $13 \%$ & 0,31 \\
\hline Triste & $18 \%$ & $7,4 \%$ & $30,4 \%$ & 0,06 \\
\hline Interesado en la música y el violín & $16 \%$ & $25,9 \%$ & $4,3 \%$ & 0,06 \\
\hline Cansado & $12 \%$ & $18,5 \%$ & $4,3 \%$ & 0,2 \\
\hline $\begin{array}{l}\text { Sin interés en la música ni en el } \\
\text { violín, no le gusta o se aburre }\end{array}$ & $10 \%$ & $11,1 \%$ & $8,7 \%$ & 1 \\
\hline $\begin{array}{l}\text { Con los brazos o las manos } \\
\text { en la cara o en la cabeza }\end{array}$ & $10 \%$ & $7,4 \%$ & $13 \%$ & 0,65 \\
\hline Preocupado & $8 \%$ & $3,7 \%$ & $13 \%$ & 0,32 \\
\hline \multicolumn{5}{|l|}{ II. ENTORNO Y OBJETOS } \\
\hline Violín & $60 \%$ & $70,4 \%$ & $47,8 \%$ & 0,15 \\
\hline Mesa & $10 \%$ & $11,1 \%$ & $8,7 \%$ & 1 \\
\hline
\end{tabular}




\section{PROBLEMAS Y SOLUCIONES}

\begin{tabular}{|l|c|c|c|c|}
\hline $\begin{array}{l}\text { A. Muchacho pensando } \\
\text { si podrá tocar el violín }\end{array}$ & $20 \%$ & $25,9 \%$ & $13 \%$ & 0,31 \\
\hline 1. No podrá & $4 \%$ & $7,4 \%$ & $0 \%$ & 0,5 \\
\hline 2. Podrá & $10 \%$ & $11,1 \%$ & $8,7 \%$ & 1 \\
\hline
\end{tabular}

\begin{tabular}{|c|c|c|c|c|}
\hline \multicolumn{5}{|l|}{ Lámina 2 "La estudiante en el campo" } \\
\hline I. FIGURAS & Total & Hombres & Mujeres & $\mathbf{p}$ \\
\hline \multicolumn{5}{|l|}{$\begin{array}{l}\text { A. Figura en primer plano } \\
\text { o a la izquierda }\end{array}$} \\
\hline \multicolumn{5}{|l|}{ 1. Sexo } \\
\hline Femenino & $98 \%$ & $96,3 \%$ & $100 \%$ & 1 \\
\hline \multicolumn{5}{|l|}{ 2. Edad } \\
\hline Joven & $10 \%$ & $0 \%$ & $21 \%$ & 0,02 \\
\hline \multicolumn{5}{|l|}{ 3. Identidad } \\
\hline Estudiante & $30 \%$ & $33,3 \%$ & $26,1 \%$ & 0,76 \\
\hline Hija de la figura de la derecha & $20 \%$ & $29,6 \%$ & $8,7 \%$ & 0,09 \\
\hline Hija de la figura en el fondo & $14 \%$ & $25,9 \%$ & $4,3 \%$ & 0,03 \\
\hline Maestra o profesora & $10 \%$ & $14,8 \%$ & $4,3 \%$ & 0,36 \\
\hline \multicolumn{5}{|l|}{ 4. Otras caracterizaciones } \\
\hline Pensando & $18 \%$ & $11,1 \%$ & $26,1 \%$ & 0,27 \\
\hline \multicolumn{5}{|l|}{ B. Figura en el fondo } \\
\hline \multicolumn{5}{|l|}{ 1. Sexo } \\
\hline Masculino & $96 \%$ & $96,3 \%$ & $95,7 \%$ & 1 \\
\hline \multicolumn{5}{|l|}{ 2. Edad } \\
\hline \multicolumn{5}{|l|}{ Sin resultados } \\
\hline \multicolumn{5}{|l|}{ 3. Identidad } \\
\hline Marido de la mujer de la derecha & $34 \%$ & $48,1 \%$ & $17,4 \%$ & 0,04 \\
\hline $\begin{array}{l}\text { Agricultor, labrador, granjero } \\
\text { o sembrador }\end{array}$ & $30 \%$ & $33,3 \%$ & $26,1 \%$ & 0,76 \\
\hline $\begin{array}{l}\text { Padre de la figura en primer plano } \\
\text { o a la izquierda }\end{array}$ & $18 \%$ & $29,6 \%$ & $4,3 \%$ & 0,03 \\
\hline
\end{tabular}




\begin{tabular}{|c|c|c|c|c|}
\hline \multicolumn{5}{|l|}{ 4. Otras caracterizaciones } \\
\hline $\begin{array}{l}\text { Labrando, arando, sembrando, } \\
\text { trillando o trabajando }\end{array}$ & $68 \%$ & $63 \%$ & $73,9 \%$ & 0,55 \\
\hline Con el torso desnudo, sin camisa & $10 \%$ & $18,5 \%$ & $0 \%$ & 0,05 \\
\hline \multicolumn{5}{|l|}{ C. Figura de la derecha } \\
\hline \multicolumn{5}{|l|}{ 1. Sexo } \\
\hline Femenino & $98 \%$ & $96,3 \%$ & $100 \%$ & 1 \\
\hline \multicolumn{5}{|l|}{ 2. Edad } \\
\hline \multicolumn{5}{|l|}{ Sin resultados } \\
\hline \multicolumn{5}{|l|}{ 3. Identidad } \\
\hline Mujer de la figura en el fondo & $34 \%$ & $48,1 \%$ & $17,4 \%$ & 0,04 \\
\hline $\begin{array}{l}\text { Madre de la figura en primer plano } \\
\text { o a la izquierda }\end{array}$ & $20 \%$ & $29,6 \%$ & $8,7 \%$ & 0,09 \\
\hline $\begin{array}{l}\text { Granjera, labradora, campesina } \\
\text { o agricultora }\end{array}$ & $18 \%$ & $18,5 \%$ & $17,4 \%$ & 1 \\
\hline \multicolumn{5}{|l|}{ 4. Otras caracterizaciones } \\
\hline $\begin{array}{l}\text { Esperando (que el hombre termine } \\
\text { de trabajar) }\end{array}$ & $18 \%$ & $14,8 \%$ & $21,7 \%$ & 0,72 \\
\hline Mirando al hombre (trabajar) & $18 \%$ & $14,8 \%$ & $21,7 \%$ & 0,72 \\
\hline Reclinada o apoyada en un árbol & $16 \%$ & $14,8 \%$ & $17,4 \%$ & 1 \\
\hline Cruzada de brazos & $14 \%$ & $18,5 \%$ & $8,7 \%$ & 0,43 \\
\hline Embarazada & $8 \%$ & $11,1 \%$ & $4,3 \%$ & 0,61 \\
\hline Mirando el horizonte o al cielo & $8 \%$ & $11,1 \%$ & $4,3 \%$ & 0,61 \\
\hline $\begin{array}{l}\text { Inactiva, pasiva, impasible, como } \\
\text { una estatua }\end{array}$ & $6 \%$ & $11,1 \%$ & $0 \%$ & 0,24 \\
\hline \multicolumn{5}{|l|}{ II. ENTORNO Y OBJETOS } \\
\hline Caballo & $78 \%$ & $85,2 \%$ & $69,6 \%$ & 0,31 \\
\hline Libros & $76 \%$ & $85,2 \%$ & $65,2 \%$ & 0,18 \\
\hline Campo o tierras & $70 \%$ & $77,8 \%$ & $60,9 \%$ & 0,23 \\
\hline Casas & $26 \%$ & $29,6 \%$ & $21,7 \%$ & 0,75 \\
\hline Montańas o monte & $24 \%$ & $18,5 \%$ & $30,4 \%$ & 0,51 \\
\hline Árbol & $20 \%$ & $14,8 \%$ & $26,1 \%$ & 0,48 \\
\hline
\end{tabular}




\begin{tabular}{|l|c|c|c|c|}
\hline Surcos & $16 \%$ & $25,9 \%$ & $4,3 \%$ & $0,06^{*}$ \\
\hline Pueblo & $10 \%$ & $14,8 \%$ & $4,3 \%$ & 0,36 \\
\hline \multicolumn{2}{|l|}{ III. PROBLEMAS Y SOLUCIONES } & \multicolumn{1}{|l|}{} \\
\hline $\begin{array}{l}\text { A. Se está esperando a que el hombre } \\
\text { termine de trabajar }\end{array}$ & $18 \%$ & $14,8 \%$ & $21,7 \%$ & 0,72 \\
\hline $\begin{array}{l}\text { B. El hombre tiene algún problema } \\
\text { con el caballo }\end{array}$ & $10 \%$ & $11,1 \%$ & $8,7 \%$ & 1 \\
\hline $\begin{array}{l}\text { C. El estudio, de alguna manera, } \\
\text { supone un conflicto }\end{array}$ & $8 \%$ & $11,1 \%$ & $4,3 \%$ & 0,61 \\
\hline \begin{tabular}{l}
1. Termina estudiando \\
\hline
\end{tabular} & $6 \%$ & $7,4 \%$ & $4,3 \%$ & 1 \\
\hline
\end{tabular}

\begin{tabular}{|l|c|}
\hline \multicolumn{2}{|c|}{ Lámina 3 VH “Reclinado/a en el diván” } \\
\hline Tiempo de reacción & Segundos \\
\hline Media & 6,15 \\
\hline DE & 4,30 \\
\hline Tiempo total & Segundos \\
\hline Media & 115,41 \\
\hline DE & 35,17 \\
\hline Número de palabras & Palabras \\
\hline Media & 134,04 \\
\hline DE & 64,12 \\
\hline
\end{tabular}

\begin{tabular}{|l|c|}
\hline Lámina 3 VH “Reclinado/a en el diván” & Hombres \\
\hline I. FIGURAS & \\
\hline A. Figura sola & \\
\hline 1. Sexo & $66,7 \%$ \\
\hline Femenino & $29,6 \%$ \\
\hline Masculino & \\
\hline 2. Edad & $18,5 \%$ \\
\hline Niño, chico, muchacho o chiquillo & \\
\hline
\end{tabular}




\begin{tabular}{|l|c|}
\hline 3. Identidad & \\
\hline Enfermo mental & $14,8 \%$ \\
\hline Vagabundo, mendigo o pobre & $11,1 \%$ \\
\hline 4. Otras caracterizaciones & \\
\hline Dormido, durmiéndose o descansando & $37 \%$ \\
\hline Llorando & $29,6 \%$ \\
\hline Sentado & $29,6 \%$ \\
\hline Pensativo o pensando & $14,8 \%$ \\
\hline Triste & $11,1 \%$ \\
\hline Disgustado & $11,1 \%$ \\
\hline II. ENTORNO Y OBJETOS & \\
\hline Banco & $25,9 \%$ \\
\hline Cama & $18,5 \%$ \\
\hline Calzado, zapatos o botas & $18,5 \%$ \\
\hline Almohada & $11,1 \%$ \\
\hline Cinturón & $11,1 \%$ \\
\hline III. PROBLEMAS Y SOLUCIONES & \\
\hline Problema amoroso o sentimental & $11,1 \%$ \\
\hline
\end{tabular}

\begin{tabular}{|l|c|}
\hline \multicolumn{2}{|c|}{ Lámina 3 NM “La joven en la puerta” } \\
\hline Tiempo de reacción & Segundos \\
\hline Media & 4,45 \\
\hline DE & 3,62 \\
\hline Tiempo total & Segundos \\
\hline Media & 80,45 \\
\hline DE & 36,93 \\
\hline Número de palabras & Palabras \\
\hline Media & 75,85 \\
\hline DE & 40,95 \\
\hline
\end{tabular}


Lámina 3 NM "La joven en la puerta”

\begin{tabular}{|l|c|}
\hline I. FIGURAS & Mujeres \\
\hline A. Figura sola & \\
\hline 1. Sexo & \\
\hline Femenino & $100 \%$ \\
\hline 2. Edad & \\
\hline Muchacha o chica & $15 \%$ \\
\hline 3. Identidad & \\
\hline Sin resultados & \\
\hline 4. Otras caracterizaciones & \\
\hline Llorando & $70 \%$ \\
\hline Con una mano en la cara o cabeza, se tapa la cara & $30 \%$ \\
\hline Con una mano en la puerta, agarrando la puerta o apoyada en la puerta & $30 \%$ \\
\hline Triste & $20 \%$ \\
\hline Disgustada o angustiada & $20 \%$ \\
\hline Pensativa & $15 \%$ \\
\hline Enferma o mareada & $15 \%$ \\
\hline Abriendo la puerta & $15 \%$ \\
\hline Cinturón & \\
\hline II. ENTORNO Y OBJETOS & \\
\hline Puerta & \\
\hline III. PROBLEMAS Y SOLUCIONES & \\
\hline Muerte de algún ser querido o desgracia familiar & \\
\hline & \\
\hline & \\
\hline & \\
\hline & \\
\hline & \\
\hline
\end{tabular}




\begin{tabular}{|c|c|c|c|c|}
\hline I. FIGURAS & Total & Hombres & Mujeres & $\mathbf{p}$ \\
\hline \multicolumn{5}{|l|}{ A. Figura a la izquierda } \\
\hline \multicolumn{5}{|l|}{ 1. Sexo } \\
\hline Masculino & $98 \%$ & $96,3 \%$ & $100 \%$ & 1 \\
\hline \multicolumn{5}{|l|}{ 2. Edad } \\
\hline \multicolumn{5}{|l|}{ Sin resultados } \\
\hline \multicolumn{5}{|l|}{ 3. Identidad } \\
\hline Marido, pareja, novio o enamorados & $56 \%$ & $63 \%$ & $47,8 \%$ & 0,39 \\
\hline Actor de cine & $32 \%$ & $37 \%$ & $26,1 \%$ & 0,55 \\
\hline \multicolumn{5}{|l|}{ 4. Otras caracterizaciones } \\
\hline $\begin{array}{l}\text { Desinteresado en la otra figura, } \\
\text { rechazándola, mirando hacia otra } \\
\text { persona u otro lado, apartándose, } \\
\text { marchándose, huyendo, alejándose }\end{array}$ & $46 \%$ & $37 \%$ & $56,5 \%$ & 0,26 \\
\hline Feliz, queriéndola o la quiere & $18 \%$ & $22,2 \%$ & $13 \%$ & 0,48 \\
\hline Violento, furioso, rabioso o enfadado & $18 \%$ & $15,5 \%$ & $17,4 \%$ & 1 \\
\hline Abrazado a la otra figura & $10 \%$ & $11,1 \%$ & $8,7 \%$ & 1 \\
\hline Triste & $6 \%$ & $11,1 \%$ & $0 \%$ & 0,24 \\
\hline Hablando & $6 \%$ & $11,1 \%$ & $0 \%$ & 0,24 \\
\hline Con bufanda o pańuelo & $6 \%$ & $11,1 \%$ & $0 \%$ & 0,24 \\
\hline \multicolumn{5}{|l|}{ B. Figura a la derecha } \\
\hline \multicolumn{5}{|l|}{ 1. Sexo } \\
\hline Femenino & $98 \%$ & $96,3 \%$ & $100 \%$ & 1 \\
\hline \multicolumn{5}{|l|}{ 2. Edad } \\
\hline \multicolumn{5}{|l|}{ Sin resultados } \\
\hline \multicolumn{5}{|l|}{ 3. Identidad } \\
\hline Mujer, pareja, novia o enamorados & $56 \%$ & $63 \%$ & $47,8 \%$ & 0,39 \\
\hline Actriz de cine & $28 \%$ & $33,3 \%$ & $21,7 \%$ & 0,53 \\
\hline
\end{tabular}




\begin{tabular}{|c|c|c|c|c|}
\hline \multicolumn{5}{|l|}{ 4. Otras caracterizaciones } \\
\hline $\begin{array}{l}\text { Interesada en la otra figura, } \\
\text { reclamándole, requiriéndole, } \\
\text { cogiéndole, le agarra, le atrae, } \\
\text { le contiene o le entretiene }\end{array}$ & $46 \%$ & $40,7 \%$ & $52,2 \%$ & 0,57 \\
\hline Feliz, queriéndole o le quiere & $22 \%$ & $22,2 \%$ & $21,7 \%$ & 1 \\
\hline Abrazada a la otra figura & $14 \%$ & $11,1 \%$ & $17,4 \%$ & 0,69 \\
\hline Hablando & $8 \%$ & $14,8 \%$ & $0 \%$ & 0,12 \\
\hline Consolando o calmando & $12 \%$ & $22,2 \%$ & $0 \%$ & 0,03 \\
\hline \multicolumn{5}{|l|}{ II. ENTORNO Y OBJETOS } \\
\hline $\begin{array}{l}\text { A. Figura humana femenina } \\
\text { en el fondo }\end{array}$ & $28 \%$ & $33,3 \%$ & $21,7 \%$ & 0,53 \\
\hline Mujer en el fondo & $16 \%$ & $11,1 \%$ & $21,7 \%$ & 0,44 \\
\hline Chica o niña en el fondo & $8 \%$ & $14,8 \%$ & $0 \%$ & 0,12 \\
\hline $\begin{array}{l}\text { Desnuda, medio desnuda, } \\
\text { semidesnuda o vistiéndose }\end{array}$ & $8 \%$ & $14,8 \%$ & $0 \%$ & 0,12 \\
\hline B. Es o parece una película & $24 \%$ & $25,9 \%$ & $21,7 \%$ & 0,75 \\
\hline $\begin{array}{l}\text { C. Cuadro, foto o imagen de chica } \\
\text { en el fondo, no verdadera persona }\end{array}$ & $10 \%$ & $14,8 \%$ & $4,3 \%$ & 0.36 \\
\hline D. Bar, cabaret o teatro & $8 \%$ & $14,8 \%$ & $0 \%$ & 0,12 \\
\hline E. Casa de citas, burdel o prostíbulo & $6 \%$ & $11,1 \%$ & $0 \%$ & 0,24 \\
\hline \multicolumn{5}{|l|}{ III. PROBLEMAS Y SOLUCIONES } \\
\hline A. No se entienden o riñen & $28 \%$ & $25,9 \%$ & $30,4 \%$ & 0,76 \\
\hline $\begin{array}{l}\text { Cada uno por su lado, se separan, } \\
\text { acaban mal }\end{array}$ & $12 \%$ & $14,8 \%$ & $8,7 \%$ & 0,67 \\
\hline $\begin{array}{l}\text { Ella pretende reconciliarse o reclama } \\
\text { su atención }\end{array}$ & $10 \%$ & $7,4 \%$ & $13 \%$ & 0,65 \\
\hline $\begin{array}{l}\text { B. Mujer tratando de evitar una } \\
\text { acción violenta o delictiva del } \\
\text { hombre, o que la defienda }\end{array}$ & $16 \%$ & $18,5 \%$ & $13 \%$ & 0,71 \\
\hline C. Mujer consuela al hombre & $8 \%$ & $14,8 \%$ & $0 \%$ & 0,12 \\
\hline
\end{tabular}




\begin{tabular}{|l|c|}
\hline \multicolumn{2}{|l|}{ Lámina 6 VH “El hijo que se va” } \\
\hline Tiempo de reacción (segundos) & Segundos \\
\hline Media & 6 \\
\hline DE & 5,27 \\
\hline Tiempo total (segundos) & Segundos \\
\hline Media & 123,63 \\
\hline DE & 52,63 \\
\hline Número de palabras & Palabras \\
\hline Media & 155,56 \\
\hline DE & 82,47 \\
\hline
\end{tabular}

\begin{tabular}{|l|c|}
\hline Lámina 6 VH "El hijo que se va" & Hombres \\
\hline I. FIGURAS & \\
\hline A. Figura de la izquierda & \\
\hline 1. Sexo & $100 \%$ \\
\hline Femenino & \\
\hline 2. Edad & $48,1 \%$ \\
\hline Mayor, anciana, vieja o de avanzada edad & \\
\hline 3. Identidad & $70,4 \%$ \\
\hline Madre del hombre & $11,1 \%$ \\
\hline Abuela del hombre & \\
\hline 4. Otras caracterizaciones & $33,3 \%$ \\
\hline Triste, apenada o pesarosa & $25,9 \%$ \\
\hline Pensando o pensativa & $18,5 \%$ \\
\hline Preocupada & $14,8 \%$ \\
\hline Hablando & $14,8 \%$ \\
\hline Con el pelo blanco o canoso & $11,1 \%$ \\
\hline Mirando por la ventana
\end{tabular}




\begin{tabular}{|l|c|}
\hline B. Figura de la derecha & \\
\hline 1. Sexo & \\
\hline Masculino & $96,3 \%$ \\
\hline 2. Edad & \\
\hline Sin resultados & \\
\hline 3. Identidad & \\
\hline Hijo de la mujer & $70,4 \%$ \\
\hline 4. Otras caracterizaciones & $33,3 \%$ \\
\hline Preocupado & $33,3 \%$ \\
\hline Con sombrero & $29,6 \%$ \\
\hline Triste & $29,6 \%$ \\
\hline Pensando, pensativo o meditando & $22,2 \%$ \\
\hline Con traje: camisa, corbata, chaqueta & \\
\hline II. ENTORNO Y OBJETOS & $18,5 \%$ \\
\hline Ventana o vidriera & $11,1 \%$ \\
\hline III. PROBLEMAS Y SOLUCIONES & \\
\hline A. Ha fallecido un familiar & \\
\hline 1. Marido de ella y padre de él & \\
\hline
\end{tabular}

\begin{tabular}{|l|c|}
\hline \multicolumn{2}{|l|}{ Lámina 7 VH "Padre e hijo" } \\
\hline Tiempo de reacción & Segundos \\
\hline Media & 9,63 \\
\hline DE & 8,64 \\
\hline Tiempo total & Segundos \\
\hline Media & 115 \\
\hline DE & 54,21 \\
\hline Número de palabras & Palabras \\
\hline Media & 121,04 \\
\hline DE & 64,75 \\
\hline
\end{tabular}




\begin{tabular}{|c|c|}
\hline I. FIGURAS & Hombres \\
\hline \multicolumn{2}{|l|}{ A. Figura de la izquierda } \\
\hline \multicolumn{2}{|l|}{ 1. Sexo } \\
\hline Masculino & $96,3 \%$ \\
\hline \multicolumn{2}{|l|}{ 2. Edad } \\
\hline Viejo, mayor o anciano & $14,8 \%$ \\
\hline Mayor que la otra figura & $11,1 \%$ \\
\hline \multicolumn{2}{|l|}{ 3.Identidad } \\
\hline Padre de la otra figura & $40,7 \%$ \\
\hline Amigo de la otra figura & $11,1 \%$ \\
\hline \multicolumn{2}{|l|}{ 4. Otras caracterizaciones } \\
\hline Hablando o susurrando & $40,7 \%$ \\
\hline De trabajo, negocios (tema económico) o futuro & $11,1 \%$ \\
\hline Con bigote & $37 \%$ \\
\hline Aconsejando o consolando & $25,9 \%$ \\
\hline Contento, feliz, unidos, cariñoso con la otra figura & $22,2 \%$ \\
\hline Bien vestido (corbata, traje, etc.) & $22,2 \%$ \\
\hline Triste & $18,5 \%$ \\
\hline Pensando o pensativo & $11,1 \%$ \\
\hline Actitud serena, desapasionada, impasible o indiferente & $11,1 \%$ \\
\hline \multicolumn{2}{|l|}{ B. Figura de la derecha } \\
\hline \multicolumn{2}{|l|}{ 1. Sexo } \\
\hline Masculino & $88,9 \%$ \\
\hline \multicolumn{2}{|l|}{ 2. Edad } \\
\hline Más joven que la otra figura & $22,2 \%$ \\
\hline Hombre joven & $11,1 \%$ \\
\hline \multicolumn{2}{|l|}{ 3. Identidad } \\
\hline Hijo de la otra figura & $40,7 \%$ \\
\hline Amigo de la otra figura & $11,1 \%$ \\
\hline
\end{tabular}




\begin{tabular}{|l|c|}
\hline 4. Otras caracterizaciones & \\
\hline Hablando o susurrando & $33,3 \%$ \\
\hline De trabajo, negocios (tema económico) o futuro & $11,1 \%$ \\
\hline Triste & $25,9 \%$ \\
\hline Pensando o pensativo & $18,5 \%$ \\
\hline Contento, feliz, unidos, cariñoso con la otra figura & $18,5 \%$ \\
\hline Crítico & $14,8 \%$ \\
\hline Bien vestido (traje, corbata, etc.) & $11,1 \%$ \\
\hline II. ENTORNO Y OBJETOS & $11,1 \%$ \\
\hline Siglo XIX & \\
\hline III. PROBLEMAS Y SOLUCIONES & $25,9 \%$ \\
\hline $\begin{array}{l}\text { A. El hombre mayor aconseja al joven, le hace reflexionar, le esclarece } \\
\text { alguna cuestión, le habla desde la experiencia, transmite sabiduría }\end{array}$ & $14,8 \%$ \\
\hline \multicolumn{2}{|l|}{ Acepta el consejo, prevalece el criterio del mayor, le convence } \\
\hline B. Piensan diferente o se enfadan & $11,1 \%$ \\
\hline
\end{tabular}

\begin{tabular}{|l|c|}
\hline \multicolumn{2}{|l|}{ Lámina 7NM “Nińa y muńeca” } \\
\hline Tiempo de reacción & Segundos \\
\hline Media & 6,57 \\
\hline DE & 5,45 \\
\hline Tiempo total & Segundos \\
\hline Media & 120,52 \\
\hline DE & 50,8 \\
\hline Número de palabras & Palabras \\
\hline Media & 113,52 \\
\hline DE & 66,46 \\
\hline
\end{tabular}




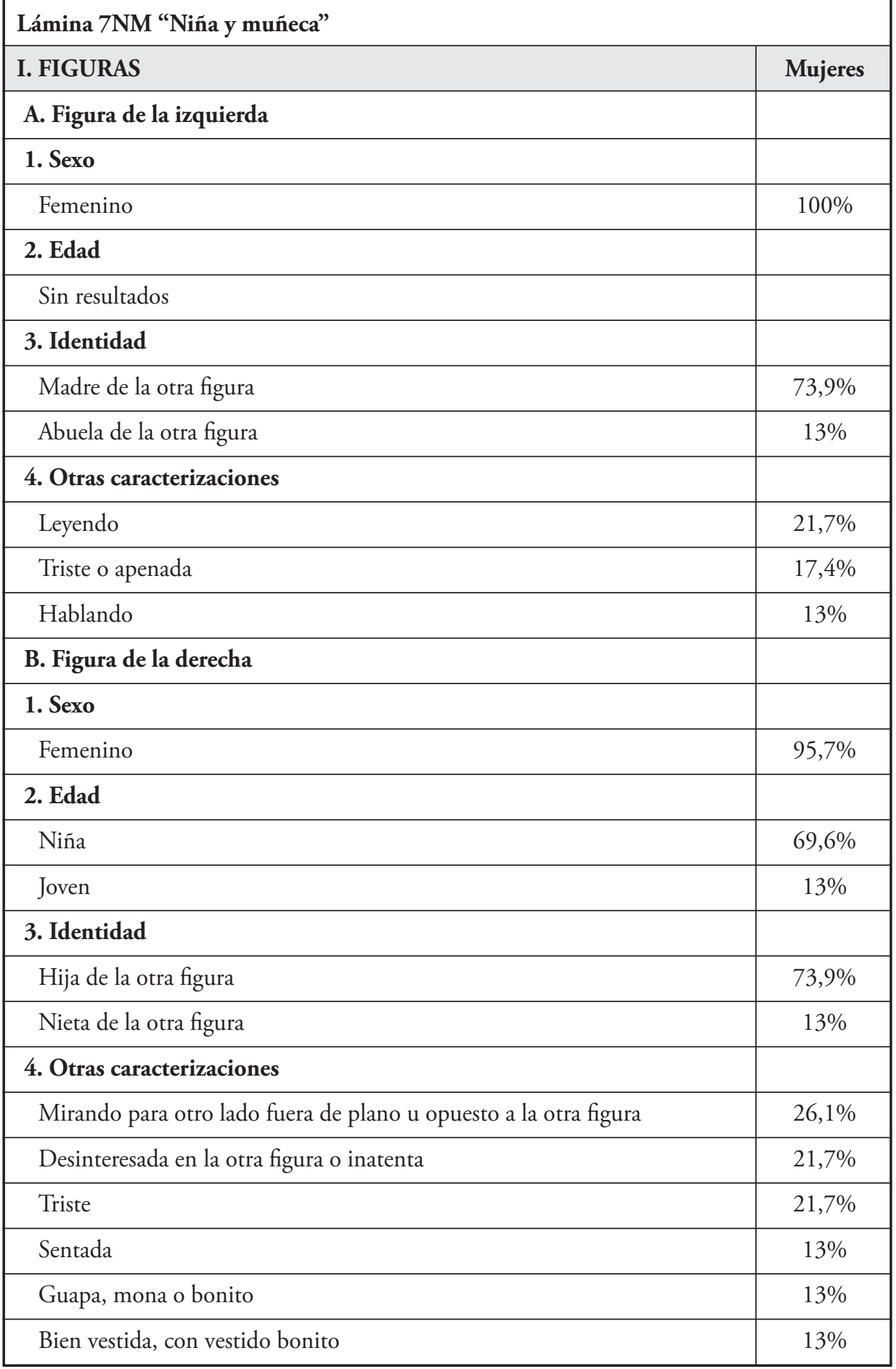




\begin{tabular}{|c|c|}
\hline Con zapatos y/o calcetines & $13 \%$ \\
\hline \multicolumn{2}{|l|}{ II. ENTORNO Y OBJETOS } \\
\hline Muñeca o muñeco (juguete) & $43,55 \%$ \\
\hline Libro & $17,4 \%$ \\
\hline Bebé o niño & $17,4 \%$ \\
\hline Sofá, sillón o tresillo & $17,4 \%$ \\
\hline Salón & $13 \%$ \\
\hline \multicolumn{2}{|c|}{ III. PROBLEMAS Y SOLUCIONES } \\
\hline No se entienden o riñen & $21,7 \%$ \\
\hline
\end{tabular}

\begin{tabular}{|l|c|}
\hline \multicolumn{2}{|l|}{ Lámina 8 VH “La intervención quirúrgica” } \\
\hline Tiempo de reacción & Segundos \\
\hline Media & 9,63 \\
\hline DE & 9,11 \\
\hline Tiempo total & Segundos \\
\hline Media & 168,63 \\
\hline DE & 71,53 \\
\hline Número de palabras & Palabras \\
\hline Media & 199,52 \\
\hline DE & 113,38 \\
\hline
\end{tabular}

\begin{tabular}{|l|c|}
\hline \multicolumn{2}{|l|}{ Lámina 8 VH “La intervención quirúrgica” } \\
\hline I. FIGURAS & Hombres \\
\hline A. Figura en primer plano & \\
\hline 1. Sexo & \\
\hline Masculino & $85,2 \%$ \\
\hline Femenino & $14,8 \%$ \\
\hline 2. Edad & \\
\hline Joven, adolescente, chico o muchacho & $48,1 \%$ \\
\hline
\end{tabular}




\begin{tabular}{|l|l|}
\hline Nińo & $14,8 \%$ \\
\hline 3. Identidad & \\
\hline Hijo de la figura echada & $22,2 \%$ \\
\hline 4. Otras caracterizaciones & \\
\hline Con traje o chaqueta & $25,9 \%$ \\
\hline Con corbata & $25,9 \%$ \\
\hline Esperando & $11,1 \%$ \\
\hline Afectado, triste o preocupado & $11,1 \%$ \\
\hline B. Figura con "cuchillo" & \\
\hline 1. Sexo & \\
\hline Masculino & $85,2 \%$ \\
\hline 2. Edad & \\
\hline Sin resultados & \\
\hline 3. Identidad & \\
\hline Doctor, médico o cirujano & $18,1 \%$ \\
\hline 4. Otras caracterizaciones & \\
\hline $\begin{array}{l}\text { Atendiendo, interviniendo, curando, auxiliando, cortando u operando } \\
\text { a la figura echada }\end{array}$ & $85,2 \%$ \\
\hline C. Figura echada en el fondo & \\
\hline 1. Sexo & \\
\hline Masculino & \\
\hline 2. Edad & \\
\hline Sin resultados & \\
\hline 3. Identidad & $22,2 \%$ \\
\hline Padre de la figura en primer plano & \\
\hline 4. Otras caracterizaciones & \\
\hline Tumbado o tendido & \\
\hline Herido & \\
\hline Muerto & \\
\hline Con dolor, sin anestesia o insuficientemente anestesiado & \\
\hline Enfermo (del estómago, del apéndice) & \\
\hline & \\
\hline & \\
\hline
\end{tabular}




\begin{tabular}{|c|c|}
\hline \multicolumn{2}{|l|}{ D. Figura con "lámpara" } \\
\hline \multicolumn{2}{|l|}{ 1. Sexo } \\
\hline Masculino & $77,8 \%$ \\
\hline \multicolumn{2}{|l|}{ 2. Edad } \\
\hline \multicolumn{2}{|l|}{ Sin resultados } \\
\hline \multicolumn{2}{|l|}{ 3. Identidad } \\
\hline Doctor, médico o cirujano & $29,6 \%$ \\
\hline Ayudante & $14,8 \%$ \\
\hline \multicolumn{2}{|l|}{ 4. Otras caracterizaciones } \\
\hline Atendiendo u operando a la figura echada & $48,1 \%$ \\
\hline Mirando o atendiendo a la figura con "cuchillo" & $14,8 \%$ \\
\hline \multicolumn{2}{|l|}{ II. ENTORNO Y OBJETOS } \\
\hline Escopeta, rifle, fusil o carabina & $48,1 \%$ \\
\hline Cuchillo, navaja o bisturí & $48,1 \%$ \\
\hline Lámpara o luz & $22,2 \%$ \\
\hline Contexto bélico, de campaña & $22,2 \%$ \\
\hline Cama o camilla & $18,5 \%$ \\
\hline Hospital & $11,1 \%$ \\
\hline \multicolumn{2}{|l|}{ III. PROBLEMAS Y SOLUCIONES } \\
\hline A. La figura echada en el fondo ha sido disparada & $40,7 \%$ \\
\hline Se recuperará o será curado & $14,8 \%$ \\
\hline $\begin{array}{l}\text { B. Independientemente del problema, la figura echada está muerta } \\
\text { o morirá }\end{array}$ & $25,9 \%$ \\
\hline C. Independientemente del problema, la figura echada se curará & $18,5 \%$ \\
\hline D. Es una autopsia & $14,8 \%$ \\
\hline $\begin{array}{l}\text { E. Están operando con una técnica inadecuada o en condiciones } \\
\text { deficientes }\end{array}$ & $14,8 \%$ \\
\hline
\end{tabular}




\begin{tabular}{|l|c|}
\hline \multicolumn{2}{|l|}{ Lámina 9 NM “Dos mujeres en la playa” } \\
\hline Tiempo de reacción & Segundos \\
\hline Media & 11,52 \\
\hline DE & 13,89 \\
\hline Tiempo total & Segundos \\
\hline Media & 128,17 \\
\hline DE & 54,23 \\
\hline Número de palabras & Palabras \\
\hline Media & 105 \\
\hline DE & 64,63 \\
\hline
\end{tabular}

\begin{tabular}{|c|c|}
\hline \multicolumn{2}{|c|}{ Lámina 9 NM "Dos mujeres en la playa” } \\
\hline I. FIGURAS & Mujeres \\
\hline \multicolumn{2}{|c|}{ A. Figura de la izquierda, abajo, fondo o segundo plano } \\
\hline \multicolumn{2}{|l|}{ 1. Sexo } \\
\hline Femenino & $91,3 \%$ \\
\hline \multicolumn{2}{|l|}{ 2. Edad } \\
\hline \multicolumn{2}{|l|}{ Sin resultados } \\
\hline \multicolumn{2}{|l|}{ 3. Identidad } \\
\hline Hija de la otra figura & $17,4 \%$ \\
\hline Amiga de la otra figura & $17,4 \%$ \\
\hline \multicolumn{2}{|l|}{ 4. Otras caracterizaciones } \\
\hline Corriendo o con prisa & $30,4 \%$ \\
\hline Huyendo o escapando & $13 \%$ \\
\hline Asustada & $13 \%$ \\
\hline \multicolumn{2}{|c|}{ B. Figura de la derecha, arriba o en primer plano } \\
\hline \multicolumn{2}{|l|}{ 1. Sexo } \\
\hline Femenino & $100 \%$ \\
\hline \multicolumn{2}{|l|}{ 2. Edad } \\
\hline Sin resultados & \\
\hline
\end{tabular}




\begin{tabular}{|l|c|}
\hline 3. Identidad & \\
\hline Madre de la otra figura & $17,4 \%$ \\
\hline Amiga de la otra figura & $17,4 \%$ \\
\hline 4. Otras caracterizaciones & $21,7 \%$ \\
\hline Corriendo o con prisa & $17,4 \%$ \\
\hline II. ENTORNO Y OBJETOS & \\
\hline $\begin{array}{l}\text { Objeto (libro o cuaderno) llevado por la mujer de la derecha, arriba o } \\
\text { en primer plano }\end{array}$ & $17,4 \%$ \\
\hline III. PROBLEMAS Y SOLUCIONES & \\
\hline Conflicto entre las dos figuras & \\
\hline
\end{tabular}

\begin{tabular}{|c|c|c|c|c|}
\hline \multicolumn{5}{|l|}{ Lámina 10 “El abrazo" } \\
\hline I. FIGURAS & Total & Hombres & Mujeres & $\mathbf{p}$ \\
\hline \multicolumn{5}{|l|}{ A. Figura a la izquierda } \\
\hline \multicolumn{5}{|l|}{ 1. Sexo } \\
\hline Masculino & $82 \%$ & $74,1 \%$ & $91,3 \%$ & 0,15 \\
\hline Femenino & $18 \%$ & $25,9 \%$ & $8,7 \%$ & 0,15 \\
\hline \multicolumn{5}{|l|}{ 2. Edad } \\
\hline \multicolumn{5}{|l|}{ Sin resultados } \\
\hline \multicolumn{5}{|l|}{ 3. Identidad } \\
\hline $\begin{array}{l}\text { Marido, mujer o pareja de la otra } \\
\text { figura }\end{array}$ & $34 \%$ & $29,6 \%$ & $39,1 \%$ & 0,56 \\
\hline Padre (o madre) de la otra figura & $20 \%$ & $18,5 \%$ & $21,7 \%$ & 1 \\
\hline Amigo o amiga de la otra figura & $12 \%$ & $11,1 \%$ & $13 \%$ & 1 \\
\hline \multicolumn{5}{|l|}{ 4. Otras caracterizaciones } \\
\hline $\begin{array}{l}\text { Amando o queriendo a la otra figura, } \\
\text { en actitud cariñosa, contento, feliz, a } \\
\text { gusto, aliviado, en paz o tranquilidad }\end{array}$ & $50 \%$ & $48,1 \%$ & $52,2 \%$ & 1 \\
\hline Besando a la otra figura (en la frente) & $40 \%$ & $40,7 \%$ & $39,1 \%$ & 1 \\
\hline Abrazado o abrazando a la otra figura & $28 \%$ & $29,6 \%$ & $26,1 \%$ & 1 \\
\hline $\begin{array}{l}\text { Hablando (al oído), susurrando o } \\
\text { cuchicheando }\end{array}$ & $16 \%$ & $18,5 \%$ & $13 \%$ & 0,71 \\
\hline
\end{tabular}




\begin{tabular}{|c|c|c|c|c|}
\hline Triste & $14 \%$ & $11,1 \%$ & $17,4 \%$ & 0,69 \\
\hline Ojos cerrados & $12 \%$ & $18,5 \%$ & $4,3 \%$ & 0,2 \\
\hline Bailando & $8 \%$ & $14,8 \%$ & $0 \%$ & 0,16 \\
\hline \multicolumn{5}{|l|}{ B. Figura a la derecha } \\
\hline \multicolumn{5}{|l|}{ 1. Sexo } \\
\hline Femenino & $58 \%$ & $63 \%$ & $52,2 \%$ & 0,57 \\
\hline Masculino & $42 \%$ & $37 \%$ & $47,8 \%$ & 0,57 \\
\hline \multicolumn{5}{|l|}{ 2. Edad } \\
\hline \multicolumn{5}{|l|}{ Sin resultados } \\
\hline \multicolumn{5}{|l|}{ 3. Identidad } \\
\hline Mujer, marido o pareja de la otra figura & $34 \%$ & $29,6 \%$ & $39,1 \%$ & 0,56 \\
\hline Hija o hijo de la otra figura & $20 \%$ & $18,5 \%$ & $21,7 \%$ & 1 \\
\hline Amiga o amigo de la otra figura & $12 \%$ & $11,1 \%$ & $13 \%$ & 1 \\
\hline \multicolumn{5}{|l|}{ 4. Otras caracterizaciones } \\
\hline $\begin{array}{l}\text { Amando o queriendo a la otra figura, } \\
\text { acariciándola, contenta, a gusto, } \\
\text { en paz o tranquilidad }\end{array}$ & $54 \%$ & $48,1 \%$ & $60,9 \%$ & 0,41 \\
\hline Abrazada o abrazando a la otra figura & $28 \%$ & $29,6 \%$ & $26,1 \%$ & 1 \\
\hline Ojos cerrados & $14 \%$ & $18,5 \%$ & $8,7 \%$ & 0,43 \\
\hline Triste & $14 \%$ & $11,1 \%$ & $17,4 \%$ & 0,69 \\
\hline $\begin{array}{l}\text { Besando a la otra figura o se están } \\
\text { besando }\end{array}$ & $12 \%$ & $11,1 \%$ & $13 \%$ & 1 \\
\hline Hablando o cuchicheando & $8 \%$ & $11,1 \%$ & $4,3 \%$ & 0,61 \\
\hline Bailando & $8 \%$ & $14,8 \%$ & $0 \%$ & 0,12 \\
\hline \multicolumn{5}{|l|}{ II. ENTORNO Y OBJETOS } \\
\hline $\begin{array}{l}\text { Mano (cualidad observada de dedos, } \\
\text { uńas...) }\end{array}$ & $30 \%$ & $29,6 \%$ & $30,4 \%$ & 1 \\
\hline Oscuridad, penumbra, negrura o sombras & $10 \%$ & $7,4 \%$ & $13 \%$ & 0,65 \\
\hline \multicolumn{5}{|l|}{ III. PROBLEMAS Y SOLUCIONES } \\
\hline Se encuentran o reencuentran & $10 \%$ & $14,8 \%$ & $4,3 \%$ & 0,38 \\
\hline Están despidiéndose & $8 \%$ & $11,1 \%$ & $4,3 \%$ & 0,61 \\
\hline Se casarán & $6 \%$ & $11,1 \%$ & $0 \%$ & 0,24 \\
\hline
\end{tabular}




\begin{tabular}{|l|c|}
\hline \multicolumn{2}{|l|}{ Lámina 12 M “La celestina” } \\
\hline Tiempo de reacción & Segundos \\
\hline Media & 6,25 \\
\hline DE & 6,35 \\
\hline Tiempo total & Segundos \\
\hline Media & 113,87 \\
\hline DE & 53,60 \\
\hline Número de palabras & Palabras \\
\hline Media & 98,74 \\
\hline DE & 58,04 \\
\hline
\end{tabular}

\begin{tabular}{|c|c|}
\hline \multicolumn{2}{|l|}{ Lámina $12 \mathrm{M}$ "La celestina" } \\
\hline I. FIGURAS & Mujeres \\
\hline \multicolumn{2}{|l|}{ A. Figura de la izquierda o en primer plano } \\
\hline \multicolumn{2}{|l|}{ 1. Sexo } \\
\hline Femenino & $87 \%$ \\
\hline Masculino & $13 \%$ \\
\hline \multicolumn{2}{|l|}{ 2. Edad } \\
\hline Joven & $13 \%$ \\
\hline \multicolumn{2}{|l|}{ 3. Identidad } \\
\hline Hija de la otra figura & $13 \%$ \\
\hline \multicolumn{2}{|l|}{ 4. Otras caracterizaciones } \\
\hline $\begin{array}{l}\text { Mirando hacia un lado, al lado opuesto a la otra figura, no la quiere mirar, } \\
\text { le desinteresa }\end{array}$ & $17,4 \%$ \\
\hline Asustada & $13 \%$ \\
\hline \multicolumn{2}{|l|}{ B. Figura de la derecha o en segundo plano } \\
\hline \multicolumn{2}{|l|}{ 1. Sexo } \\
\hline Femenino & $95,7 \%$ \\
\hline \multicolumn{2}{|l|}{ 2. Edad } \\
\hline Mayor o vieja & $34,8 \%$ \\
\hline
\end{tabular}




\begin{tabular}{|l|c|}
\hline 3. Identidad & \\
\hline Bruja o hechicera & $34,8 \%$ \\
\hline Madre de la otra figura & $13 \%$ \\
\hline 4. Otras caracterizaciones & \\
\hline Feliz o contenta & $17,4 \%$ \\
\hline Con arrugas & $13 \%$ \\
\hline II. ENTORNO Y OBJETOS & $17,4 \%$ \\
\hline Es un cuadro & \\
\hline III. PROBLEMAS Y SOLUCIONES & $13 \%$ \\
\hline La figura derecha (bruja) trata de perjudicar a la otra figura & \\
\hline
\end{tabular}

\begin{tabular}{|c|c|c|c|c|}
\hline \multicolumn{5}{|l|}{ Lámina 13 HM "Mujer en la cama" } \\
\hline I. FIGURAS & Total & Hombres & Mujeres & $\mathbf{p}$ \\
\hline \multicolumn{5}{|l|}{ A. Figura a la izquierda } \\
\hline \multicolumn{5}{|l|}{ 1. Sexo } \\
\hline Femenino & $98 \%$ & $96,3 \%$ & $100 \%$ & 1 \\
\hline \multicolumn{5}{|l|}{ 2. Edad } \\
\hline \multicolumn{5}{|l|}{ Sin resultados } \\
\hline \multicolumn{5}{|l|}{ 3.Identidad } \\
\hline Mujer o pareja de la otra figura & $64 \%$ & $55,6 \%$ & $73,9 \%$ & 0,24 \\
\hline Amiga de la otra figura & $8 \%$ & $11,1 \%$ & $4,3 \%$ & 0,61 \\
\hline \multicolumn{5}{|l|}{ 4. Otras caracterizaciones } \\
\hline $\begin{array}{l}\text { Desnuda, semidesnuda o medio } \\
\text { desnuda, se le ven los pechos } \\
\text { o los enseńa }\end{array}$ & $46 \%$ & $51,9 \%$ & $39,1 \%$ & 0,41 \\
\hline Muerta & $42 \%$ & $40,7 \%$ & $43,5 \%$ & 1 \\
\hline Echada o tumbada & $26 \%$ & $33,3 \%$ & $17,4 \%$ & 0,33 \\
\hline Enferma & $22 \%$ & $11,1 \%$ & $34,8 \%$ & 0,08 \\
\hline Durmiendo, descansando o relajada & $14 \%$ & $22,2 \%$ & $4,3 \%$ & 0,11 \\
\hline Con un brazo (o los brazos) caído(s) & $12 \%$ & $14,8 \%$ & $8,7 \%$ & 0,67 \\
\hline
\end{tabular}




\begin{tabular}{|c|c|c|c|c|}
\hline $\begin{array}{l}\text { Triste, decaída, afectada, } \\
\text { descompuesta o hecha polvo }\end{array}$ & $10 \%$ & $7,4 \%$ & $13 \%$ & 0,65 \\
\hline \multicolumn{5}{|l|}{ B. Figura a la derecha } \\
\hline \multicolumn{5}{|l|}{ 1. Sexo } \\
\hline Masculino & $100 \%$ & $100 \%$ & $100 \%$ & \\
\hline \multicolumn{5}{|l|}{ 2. Edad } \\
\hline \multicolumn{5}{|l|}{ Sin resultados } \\
\hline \multicolumn{5}{|l|}{ 3. Identidad } \\
\hline Marido o pareja de la otra figura & $64 \%$ & $55,6 \%$ & $73,9 \%$ & 0,24 \\
\hline Amigo de la otra figura & $8 \%$ & $11,1 \%$ & $4,3 \%$ & 0,61 \\
\hline \multicolumn{5}{|l|}{ 4. Otras caracterizaciones } \\
\hline $\begin{array}{l}\text { Se tapa los ojos o la cara, se tapa } \\
\text { para no ver o no quiere ver }\end{array}$ & $42 \%$ & $44,4 \%$ & $39,1 \%$ & 0,78 \\
\hline Llorando & $42 \%$ & $33,3 \%$ & $52,2 \%$ & 0,25 \\
\hline $\begin{array}{l}\text { Vestido o bien vestido } \\
\text { (corbata, traje...) }\end{array}$ & $38 \%$ & $44,4 \%$ & $30,4 \%$ & 0,39 \\
\hline $\begin{array}{l}\text { Arrepentido, abatido, culpable, } \\
\text { lamentándose, entristecido } \\
\text { o apenado }\end{array}$ & $28 \%$ & $33,3 \%$ & $21,7 \%$ & 0,53 \\
\hline De pie & $12 \%$ & $22,2 \%$ & $0 \%$ & 0,03 \\
\hline Preocupado & $10 \%$ & $18,5 \%$ & $0 \%$ & 0,05 \\
\hline \multicolumn{5}{|l|}{ II. ENTORNO Y OBJETOS } \\
\hline Libros & $60 \%$ & $59,3 \%$ & $60,9 \%$ & 1 \\
\hline Cama, camilla, camastro, lecho o catre & $58 \%$ & $59,3 \%$ & $56,5 \%$ & 1 \\
\hline Mesa o mesilla & $50 \%$ & $51,9 \%$ & $47,8 \%$ & 1 \\
\hline Cuadro & $48 \%$ & $44,4 \%$ & $52,2 \%$ & 0,78 \\
\hline Lámpara & $42 \%$ & $33,3 \%$ & $52,2 \%$ & 0,25 \\
\hline Habitación, dormitorio o cuarto & $26 \%$ & $25,9 \%$ & $26,1 \%$ & 1 \\
\hline Silla & $22 \%$ & $29,6 \%$ & $13 \%$ & 0,19 \\
\hline Manta o sábana & $8 \%$ & $14,8 \%$ & $0 \%$ & 0,16 \\
\hline \multicolumn{5}{|l|}{ III. PROBLEMAS Y SOLUCIONES } \\
\hline A. El hombre ha matado a la mujer & $20 \%$ & $22,2 \%$ & $17,4 \%$ & 0,74 \\
\hline
\end{tabular}




\begin{tabular}{|l|c|c|c|c|}
\hline $\begin{array}{l}\text { 1. Se entregará o será detenido y/o } \\
\text { juzgado-castigado }\end{array}$ & $12 \%$ & $14,8 \%$ & $8,7 \%$ & 0,67 \\
\hline 2. Por infiel, celos o asuntos amorosos & $10 \%$ & $11,1 \%$ & $8,7 \%$ & 1 \\
\hline $\begin{array}{l}\text { B. La mujer ha muerto, está } \\
\text { muriéndose o morirá } \\
\text { por enfermedad }\end{array}$ & $16 \%$ & $11,1 \%$ & $21,7 \%$ & 0,44 \\
\hline $\begin{array}{l}\text { C. La relación sexual entre ambas } \\
\text { figuras o el deseo sexual (o la } \\
\text { ausencia del mismo) de alguna de } \\
\text { las figuras supone un conflicto }\end{array}$ & $14 \%$ & $14,8 \%$ & $13 \%$ & 1 \\
\hline $\begin{array}{l}\text { D. El hombre se marcha (a trabajar } \\
\text { o por cualquier otro motivo) }\end{array}$ & $14 \%$ & $14,8 \%$ & $13 \%$ & 1 \\
\hline $\begin{array}{l}\text { E. El hombre no quiere a la mujer } \\
\text { o hay algún conflicto entre ambos } \\
\text { de otra índole no recogida en } \\
\text { otras causas }\end{array}$ & $6 \%$ & $0 \%$ & $13 \%$ & 0,09 \\
\hline
\end{tabular}

\section{Lámina 14 "El hombre en la ventana"}

\begin{tabular}{|c|c|c|c|c|}
\hline I. FIGURAS & Total & Hombres & Mujeres & $\mathbf{p}$ \\
\hline \multicolumn{5}{|l|}{ A. Figura sola } \\
\hline \multicolumn{5}{|l|}{ 1. Sexo } \\
\hline Masculino & $84 \%$ & $92,6 \%$ & $73,9 \%$ & 0,12 \\
\hline Femenino & $14 \%$ & $7,4 \%$ & $21,7 \%$ & 0,23 \\
\hline \multicolumn{5}{|l|}{ 2. Edad } \\
\hline \multicolumn{5}{|l|}{ Sin resultados } \\
\hline \multicolumn{5}{|l|}{ 3. Identidad } \\
\hline Preso, recluso, encerrado o entre rejas & $12 \%$ & $11,1 \%$ & $13 \%$ & 1 \\
\hline \multicolumn{5}{|l|}{ 4. Otras caracterizaciones } \\
\hline $\begin{array}{l}\text { Mirando por o asomado a la ventana } \\
\text { o puerta, o mirando hacia fuera, } \\
\text { al exterior, a la calle }\end{array}$ & $28 \%$ & $29,6 \%$ & $26,1 \%$ & 1 \\
\hline $\begin{array}{l}\text { Abriendo, abre o ha abierto } \\
\text { la ventana o puerta }\end{array}$ & $22 \%$ & $25,9 \%$ & $17,4 \%$ & 0,52 \\
\hline Contento, a gusto, feliz o alegre & $18 \%$ & $18,5 \%$ & $17,4 \%$ & 1 \\
\hline Mirando al cielo & $10 \%$ & $11,1 \%$ & $8,7 \%$ & 1 \\
\hline
\end{tabular}




\begin{tabular}{|c|c|c|c|c|}
\hline Sentado & $8 \%$ & $3,7 \%$ & $13 \%$ & 0,32 \\
\hline Desesperado o siente malestar & $6 \%$ & $0 \%$ & $13 \%$ & 0,09 \\
\hline \multicolumn{5}{|l|}{ II. ENTORNO Y OBJETOS } \\
\hline Ventana & $84 \%$ & $81,5 \%$ & $87 \%$ & 0,71 \\
\hline $\begin{array}{l}\text { Oscuridad, sombra, negrura, } \\
\text { tenebroso o ausencia de luz }\end{array}$ & $56 \%$ & $66,7 \%$ & $43,5 \%$ & 0,15 \\
\hline Luz o claridad & $22 \%$ & $33,3 \%$ & $8,7 \%$ & 0,046 \\
\hline Habitación o cuarto & $18 \%$ & $22,2 \%$ & $13 \%$ & 0,48 \\
\hline De noche o anocheciendo & $16 \%$ & $14,8 \%$ & $17,4 \%$ & 1 \\
\hline Puerta & $14 \%$ & $14,8 \%$ & $13 \%$ & 1 \\
\hline \multicolumn{5}{|l|}{ III. PROBLEMAS Y SOLUCIONES } \\
\hline $\begin{array}{l}\text { A. Figura pensando en la libertad } \\
\text { o que quiere huir, escapar o salir } \\
\text { del entorno, de una situación } \\
\text { problemática, o de la negrura en la } \\
\text { que se encuentra (hacia la luz) }\end{array}$ & $30 \%$ & $33,3 \%$ & $26,1 \%$ & 0,76 \\
\hline Huirá, escapará o saldrá & $26 \%$ & $29,6 \%$ & $21,7 \%$ & 0,75 \\
\hline $\begin{array}{l}\text { B. Figura pensando en suicidarse } \\
\text { o tirarse por la ventana, o quiere } \\
\text { suicidarse }\end{array}$ & $18 \%$ & $18,5 \%$ & $17,4 \%$ & 1 \\
\hline No se suicidará o no se tirará & $14 \%$ & $11,1 \%$ & $17,4 \%$ & 0,69 \\
\hline
\end{tabular}

\begin{tabular}{|l|c|}
\hline \multicolumn{2}{|l|}{ Lámina $\mathbf{1 8}$ VH “Atacado desde atrás” } \\
\hline Tiempo de reacción & Segundos \\
\hline Media & 9,70 \\
\hline DE & 6,56 \\
\hline Tiempo total & Segundos \\
\hline Media & 118,74 \\
\hline DE & 36,95 \\
\hline Número de palabras & Palabras \\
\hline Media & 124,15 \\
\hline DE & 61,92 \\
\hline
\end{tabular}




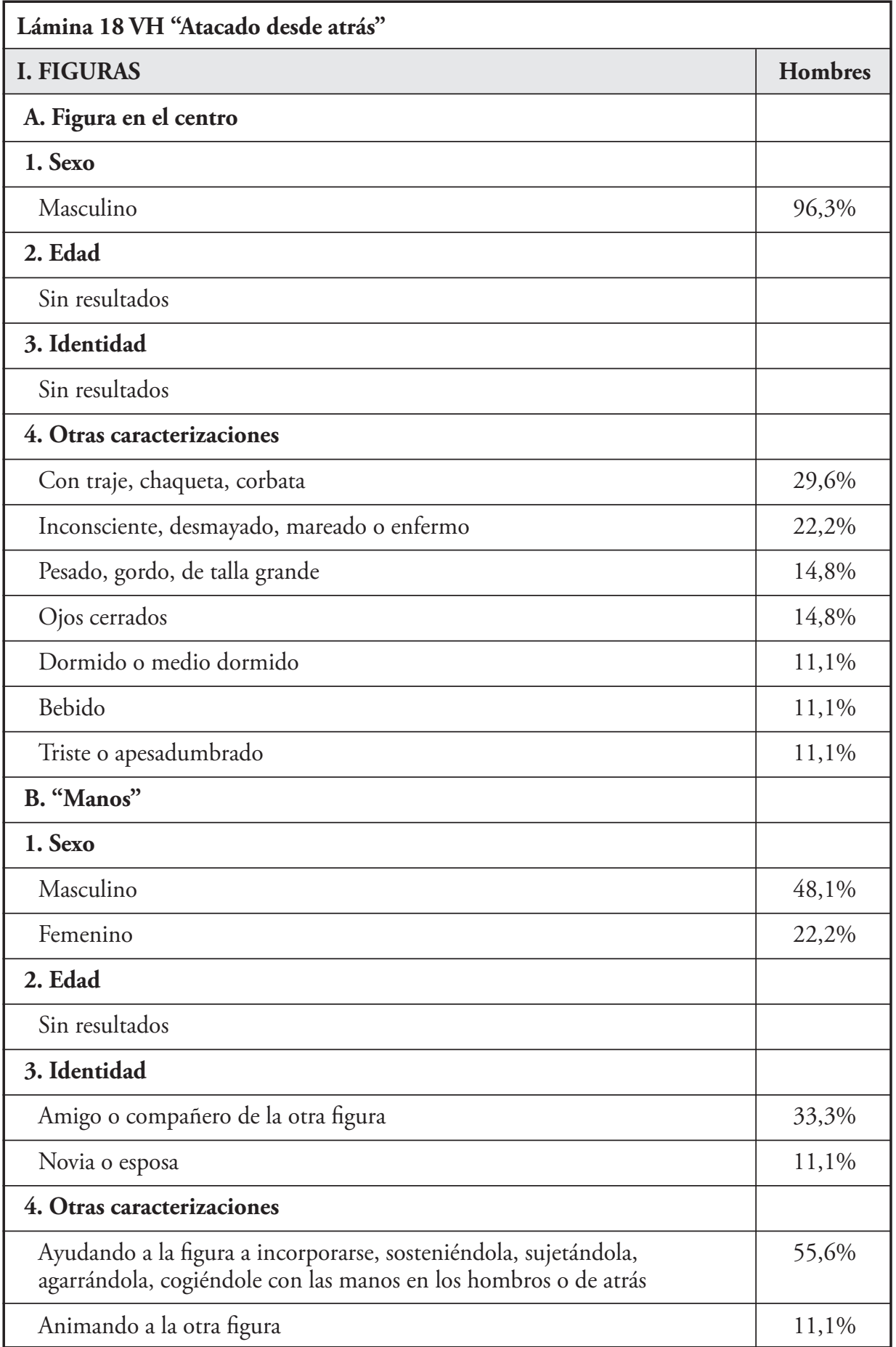




\section{ENTORNO Y OBJETOS}

Oscuro

$11,1 \%$

III. PROBLEMAS Y SOLUCIONES

De alguna forma las manos ayudan a la figura

$33,3 \%$

Figura inconsciente, desmayada, mareada o enferma, a la que las "manos" están auxiliando

Hombre alcoholizado

\begin{tabular}{|l|c|}
\hline \multicolumn{2}{|l|}{ Lámina 18 NM “Mujer que estrangula” } \\
\hline Tiempo de reacción & Segundos \\
\hline Media & 7,48 \\
\hline DE & 4,18 \\
\hline Tiempo total & Segundos \\
\hline Media & 122,17 \\
\hline DE & 35,2 \\
\hline Número de palabras & Palabras \\
\hline Media & 109,13 \\
\hline DE & 51,53 \\
\hline
\end{tabular}

Lámina 18 NM "Mujer que estrangula"

\begin{tabular}{|l|c|}
\hline I. FIGURAS & Mujeres \\
\hline A. Figura de la izquierda & \\
\hline 1. Sexo & $82,6 \%$ \\
\hline Femenino & $17,4 \%$ \\
\hline Masculino & \\
\hline 2. Edad & $13 \%$ \\
\hline Mayor & \\
\hline 3. Identidad & $39,1 \%$ \\
\hline Madre de la otra figura & \\
\hline
\end{tabular}




\begin{tabular}{|c|c|}
\hline Hijo o hija de la otra figura & $21,7 \%$ \\
\hline Hermana de la otra figura & $13 \%$ \\
\hline \multicolumn{2}{|l|}{ 4. Otras caracterizaciones } \\
\hline Enferma, mareada o desvanecida & $30,4 \%$ \\
\hline \multicolumn{2}{|l|}{ B. Figura de la derecha } \\
\hline \multicolumn{2}{|l|}{ 1. Sexo } \\
\hline Femenino & $95,7 \%$ \\
\hline \multicolumn{2}{|l|}{ 2. Edad } \\
\hline Niña & $21,7 \%$ \\
\hline \multicolumn{2}{|l|}{ 3. Identidad } \\
\hline Hija de la otra figura & $39,1 \%$ \\
\hline Madre de la otra figura & $21,7 \%$ \\
\hline Hermana de la otra figura & $13 \%$ \\
\hline \multicolumn{2}{|l|}{ 4. Otras caracterizaciones } \\
\hline Agarrando, sosteniendo, manteniendo, cogiendo o sujetando a la otra figura & $47,8 \%$ \\
\hline Llorando & $43,5 \%$ \\
\hline Triste & $39,1 \%$ \\
\hline Mira o contempla a la otra figura & $13 \%$ \\
\hline \multicolumn{2}{|l|}{ II. ENTORNO Y OBJETOS } \\
\hline Escalera & $56,5 \%$ \\
\hline \multicolumn{2}{|l|}{ III. PROBLEMAS Y SOLUCIONES } \\
\hline $\begin{array}{l}\text { A. La figura derecha encuentra, acude, da ayuda o consuelo } \\
\text { en enfermedad, dańo o susto a la otra figura }\end{array}$ & $82,6 \%$ \\
\hline 1. La otra figura morirá & $39,1 \%$ \\
\hline 2. Se ha caído por las escaleras & $21,7 \%$ \\
\hline 3. La otra figura mejorará & $21,7 \%$ \\
\hline
\end{tabular}

